The Astrophysical Journal, 669:1220-1234, 2007 November 10

(C) 2007. The American Astronomical Society. All rights reserved. Printed in U.S.A.

\title{
THE METALLICITY OF STARS WITH CLOSE COMPANIONS
}

\author{
Daniel Grether ${ }^{1}$ and Charles H. Lineweaver ${ }^{2}$ \\ Received 2006 November 30; accepted 2007 July 5
}

\begin{abstract}
We examine the relationship between the frequency of close companions (stellar and planetary companions with orbital periods $<5 \mathrm{yr}$ ) and the metallicity of their Sun-like ( $\sim$ FGK) hosts. We confirm and quantify a $\sim 4 \sigma$ positive correlation between host metallicity and planetary companions. We find little or no dependence on spectral type or distance in this correlation. In contrast to the metallicity dependence of planetary companions, stellar companions tend to be more abundant around low-metallicity hosts. At the $\sim 2 \sigma$ level, we find an anticorrelation between host metallicity and the presence of a stellar companion. After dividing our sample into FG and $\mathrm{K}$ subsamples, we find a negligible anticorrelation in the FG subsample and $\mathrm{a} \sim 3 \sigma$ anticorrelation in the $\mathrm{K}$ subsample. A kinematic analysis suggests that this anticorrelation is produced by a combination of low-metallicity, high-binarity thick-disk stars and higher metallicity, lower binarity thin-disk stars.
\end{abstract}

Subject headings: binaries: close — stars: abundances — stars: kinematics

\section{INTRODUCTION}

With the detection to date of more than 160 exoplanets using the Doppler technique, the observation of Gonzalez (1997) that giant close-orbiting exoplanets have host stars with relatively high stellar metallicity compared to the average field star has gotten stronger (Reid 2002; Santos et al. 2004; Fischer \& Valenti 2005; Bond et al. 2006). To understand the nature of this correlation between high host metallicity and the presence of Doppler-detectable exoplanets, we investigate whether this correlation extends to stellar mass companions.

There has been a widely held view that metal-poor stellar populations possess few stellar companions (Batten 1973; Latham et al. 1988; Latham 2004). This may have been largely due to the difficulty of finding binary stars in the Galactic halo (e.g., Gunn \& Griffin 1979). Duquennoy \& Mayor (1991) investigated the properties of stellar companions among Sun-like stars, but did not report a relationship between stellar companions and host metallicity. Latham et al. (2002) and Carney et al. (2005) reported a lower binarity for stars on retrograde Galactic orbits compared to stars on prograde Galactic orbits, but found no dependence between binarity and metallicity within those two kinematic groups. Dall et al. (2005) speculated that the frequency of host stars with stellar companions may be correlated with metallicity in the same way that host stars with planets are.

In this paper we describe and characterize the correlation between host metallicity and the fraction of planetary and stellar companions. In $\S 2$ we define our sample of close planetary and stellar companions, and we describe the variety of techniques used to obtain metallicities of stars that do not have spectroscopic metallicities from Doppler searches. In $\S 3$ we analyze the distribution of planetary and stellar companions as a function of host metallicity. We confirm and quantify the correlation between planet hosts and high metallicity, and we find a new anticorrelation between the frequency of stellar companions and high metallicity. In $\S 4$ we compare our stellar companion results to analogous

\footnotetext{
1 Department of Astrophysics, School of Physics, University of New South Wales, Sydney, NSW 2052, Australia.

2 Planetary Science Institute, Research School of Astronomy and Astrophysics, and Research School of Earth Sciences, Australian National University, Canberra, ACT, Australia.
}

analyses of the Nordström et al. (2004) and Carney et al. (2005) samples.

\section{THE SAMPLE}

We analyze the distribution of the metallicities of FGK mainsequence stars with close companions (period $<5 \mathrm{yr}$ ). For this we use the sample of stars analyzed by Grether \& Lineweaver (2006). This subset of Sun-like stars in the Hipparcos catalog is defined by $0.5 \leq B-V \leq 1.0$ and $5.4(B-V)+2.0 \leq M_{V} \leq$ $5.4(B-V)-0.5$. This forms a parallelogram with sides -0.5 and $2.0 \mathrm{mag}$, below and above an average main sequence in the $\mathrm{H}-\mathrm{R}$ diagram. The stars range in spectral type from approximately F7 to $\mathrm{K} 3$ and in absolute magnitude in the $V$ band from 2.2 to 7.4 . From this we define a closer, more complete $(d<25 \mathrm{pc})$ sample of stars and a more distant, independent $(25<d<50 \mathrm{pc})$ sample. See Grether \& Lineweaver (2006) for additional details about the sample definition.

\subsection{Measuring Stellar Metallicity}

The metallicity of most of the extrasolar planet hosts have been determined spectroscopically. We analyze the metallicity data from three of these groups: (1) the McDonald observatory group (McD; e.g., Gonzalez et al. 2001; Laws et al. 2003), (2) the European Southern Observatory group (ESO; e.g., Santos et al. 2004, 2005), and (3) the Keck, Lick, and Anglo-Australian observatory group (KLA; Fischer \& Valenti 2005; Valenti \& Fischer 2005).

All three of these groups find similar metallicities for the extrasolar planet target stars that they have all observed, as shown by the comparisons in Figure 1. Apart from the 1000 KLA target stars analyzed with a consistently high precision by Valenti \& Fischer (2005), many nearby $(d<50 \mathrm{pc})$ FGK stars lack precise metallicities, if they have any published measurement at all. A smaller sample of precise spectroscopic metallicities has also been published by the ESO group for non-planet-hosting stars (Santos et al. 2005).

Since the large sample of KLA stars has been taken from exoplanet target lists, it also has the same biases. This includes selection effects (1) against high stellar chromospheric activity, (2) toward more metal-rich stars that have a greater probability of being a planetary host, and (3) against most stars with known close $\left(\theta<2^{\prime \prime}\right)$ stellar companions. We need to correct for or minimize 


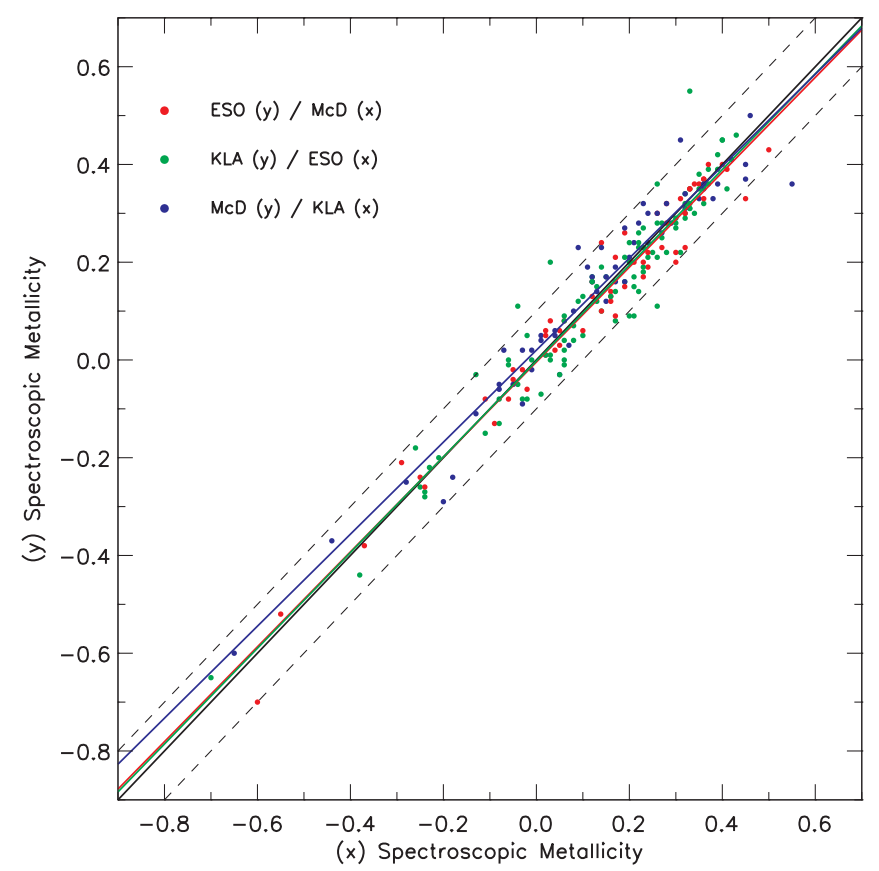

FIG. 1.-Exoplanet target stars metallicity comparison. We compare the spectroscopic exoplanet target metallicities of the McD, ESO, and KLA groups. The 59 red dots compare the ESO to the McD values of exoplanet target metallicity that these groups have in common. We find that the ESO values are on average $0.01 \mathrm{dex}$ smaller than the McD values, with a dispersion of 0.05 dex. Similarly, the 99 green dots compare the KLA values to the average 0.01 dex smaller ESO values, with a dispersion of $0.06 \mathrm{dex}$. The 56 blue dots compare the KLA to the average 0.01 dex larger $\mathrm{McD}$ values, with a dispersion of $0.06 \mathrm{dex}$. A solid black line shows the slope 1 line with dashed lines at \pm 0.1 dex. The three linear best fits for these three comparisons are nearly identical to the slope 1 line, and almost all scatter is contained within $0.1 \mathrm{dex}$. The relationship between the $\mathrm{McD}$ and ESO values is very close, with a marginally looser relationship with the KLA values. Thus, these values for exoplanet target metallicity are consistent at the $\sim 0.1$ dex level.

these biases to determine quantitatively not only how the planetary distribution varies with host metallicity but also how the close stellar companion distribution varies with host metallicity; that is, we need metallicities of all stars in our sample in order to compare companion-hosting stars to non-companion-hosting stars and to compare the metallicities of planet-hosting stars to the metallicities of stellar companion-hosting stars.

In addition to the metallicities reported by the McD, ESO, and KLA groups, we use a variety of other sources and techniques to determine stellar metallicity, although with somewhat less precision. These include other sources of spectroscopic metallicities, such as the Cayrel de Strobel et al. (2001, hereafter CdS) catalog, metallicities derived from uvby narrowband photometry or broadband photometry, and metallicities derived from a star's position in the H-R diagram. The precision of the spectroscopic metallicity values in the $\mathrm{CdS}$ catalog are not well quantified. However, many of the stars in the catalog have several independent metallicity values, which we average, excluding obvious outliers. To derive metallicities from $u v b y$ narrowband photometry, we apply the calibration of Martell \& Smith (2004) to the Hauck \& Mermilliod (1998) catalog. We also use values of metallicity derived from broadband photometry (Ammons et al. 2006). For stars with $5.5<M_{V}<7.3$ ( $\mathrm{K}$ dwarfs) the relationship between stellar luminosity and metallicity is very tight (Kotoneva et al. 2002). Using this relationship, we derive metallicities for some $\mathrm{K}$ dwarfs from their position in the H-R diagram.

To quantify the precision of their metallicities, we compare in Figure 2 the different methods of determining metallicity. We use

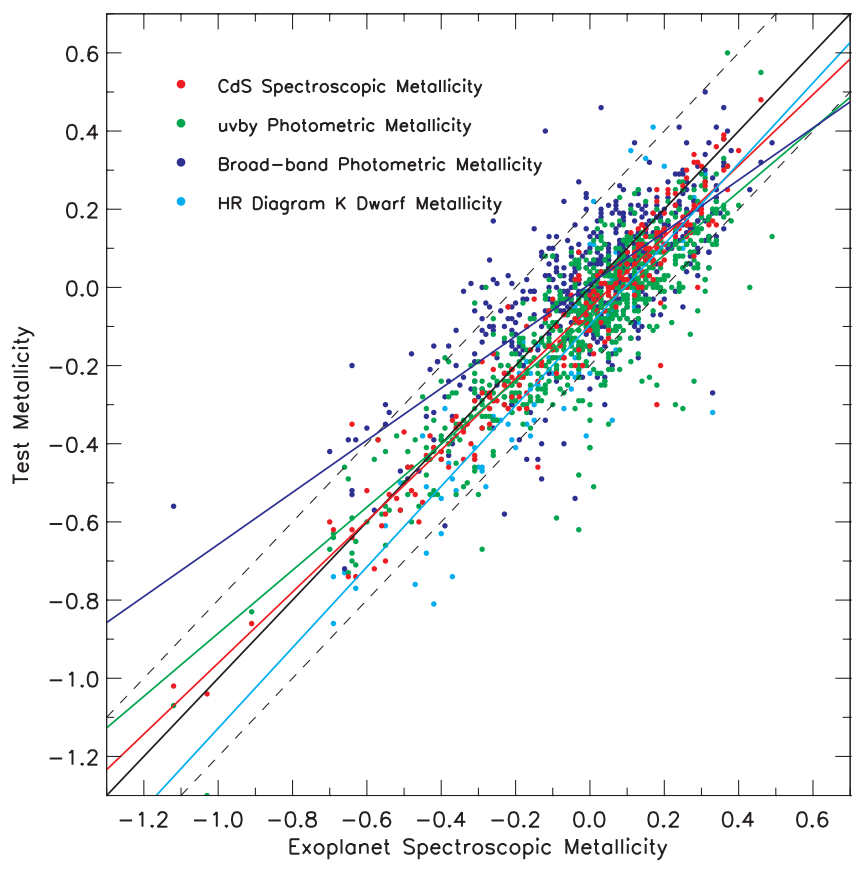

FIG. 2.-Metallicity values from exoplanet spectroscopy compared to four other methods of obtaining stellar metallicities. We compare the exoplanet target spectroscopic metallicities (plotted on the $x$-axis as a reference) with the following test samples plotted on the $y$-axis: (1) CdS spectroscopic metallicities (red dots), (2) uvby photometric metallicities ( green dots), (3) broadband photometric metallicities (blue dots), and (4) H-R diagram K dwarf metallicities (aqua dots). The mean differences between the test and the reference sample metallicities $\left([\mathrm{Fe} / \mathrm{H}]_{\text {test }}-\right.$ $\left.[\mathrm{Fe} / \mathrm{H}]_{\mathrm{ref}}\right)$ are $-0.05,-0.08,0.01$, and $-0.10 \mathrm{dex}$, respectively, with dispersions of $0.08,0.11,0.14$, and 0.14 dex, respectively. Comparing these mean differences and dispersions, we find that the mean differences are within $1 \sigma$ of the solid black slope 1 line, and thus we regard the systematic offsets as marginal. The four linear best fits for these four comparisons (shown by the four colored lines) do not show significant deviation from the slope 1 line (black), except for the metallicities derived using broadband photometry (dark blue line).

the high-precision exoplanet target spectroscopic metallicities from the $\mathrm{McD}$, ESO, and KLA surveys (or the average, when a star has two or more values) as the reference sample. We compare these metallicities with metallicities of the following test samples: (1) CdS spectroscopic metallicities, (2) uvby photometric metallicities, (3) broadband photometric metallicities, and (4) H-R diagram $\mathrm{K}$ dwarf metallicities.

The result of this comparison is that the uncertainties associated with the high-quality exoplanet target spectroscopic metallicities of the McD, ESO, and KLA groups are the smallest, with the CdS spectroscopic metallicities only slightly more uncertain. The uncertainties associated with the uvby photometric metallicities are intermediate, with broadband photometric and H-R diagram $\mathrm{K}$ dwarf metallicities being the least certain.

\subsection{Selection Effects and Completeness}

To minimize the scatter in the measurement of stellar metallicity while including as many stars in our samples as possible, we choose the metallicity source from one of the five groups based on minimal dispersion. Thus, we primarily use the spectroscopic exoplanet target metallicities. If no such value for metallicity is available for a star in our sample, we use a spectroscopic value taken from the CdS catalog, followed by a uvby photometric value, a broadband value, and, lastly, a H-R diagram $\mathrm{K}$ dwarf value for the metallicity. We use the dispersions discussed above as estimates of the uncertainties of the metallicity measurements. 
TABLE 1

Stellar Samples Used in Our Analysis

\begin{tabular}{|c|c|c|c|c|c|c|c|}
\hline \multirow[b]{2}{*}{ SAMPLE } & \multirow[b]{2}{*}{$B-V$} & \multirow{2}{*}{$\begin{array}{c}\text { RANGE } \\
(\mathrm{pc})\end{array}$} & \multicolumn{5}{|c|}{ Stars with $[\mathrm{Fe} / \mathrm{H}]$ Measurements } \\
\hline & & & Total $^{\mathrm{a}}$ & Binary $^{b}$ & Targets $^{c}$ & Planet Hosts ${ }^{\mathrm{d}}$ & {$[\mathrm{Fe} / \mathrm{H}]$ Source } \\
\hline \multirow[t]{2}{*}{ Our FGK. } & $0.5-1.0$ & $d<25$ & 453 & $45(9.9 \%)$ & $379(84 \%)$ & $19(5.0 \%)$ & Mostly spectroscopy ${ }^{\mathrm{e}}$ \\
\hline & $0.5-1.0$ & $25<d<50$ & 2745 & $107(3.9 \%)$ & $1597(58 \%)$ & $36(2.3 \%)$ & Mostly photometry \\
\hline \multirow[t]{2}{*}{ Our FG............................ } & $0.5-0.75$ & $d<25$ & 257 & $27(10.5 \%)$ & $228(89 \%)$ & $13(5.7 \%)$ & Mostly spectroscopy ${ }^{g}$ \\
\hline & $0.5-0.75$ & $25<d<50$ & 1762 & $76(4.3 \%)$ & $1167(66 \%)$ & $32(2.7 \%)$ & Mostly photometry $^{\text {h }}$ \\
\hline \multirow[t]{2}{*}{ Our K ............................. } & $0.75-1.0$ & $d<25$ & 196 & $18(9.2 \%)$ & $151(77 \%)$ & $6(4.0 \%)$ & Mostly spectroscopy ${ }^{\mathrm{i}}$ \\
\hline & $0.75-1.0$ & $25<d<50$ & 983 & $31(3.2 \%)$ & $430(44 \%)$ & $4(0.9 \%)$ & Mostly photometry ${ }^{\mathrm{j}}$ \\
\hline \multirow[t]{2}{*}{$\mathrm{GC}^{\mathrm{k}}$ FGK. } & $0.3-1.0$ & $d<40$ & 1375 & $378(27.5 \%)$ & $\ldots$ & $\ldots$ & uvby photometry \\
\hline & $0.5-1.0$ & $d<40$ & 1289 & $346(26.8 \%)$ & $\ldots$ & $\ldots$ & uvby photometry \\
\hline GC FG........................... & $0.5-0.75$ & $d<40$ & 1117 & $291(26.1 \%)$ & $\ldots$ & $\ldots$ & uvby photometry \\
\hline GC K ............................. & $0.75-1.0$ & $d<40$ & 172 & $55(32.0 \%)$ & $\ldots$ & $\ldots$ & uvby photometry \\
\hline $\mathrm{CL}^{1} \mathrm{AFGK} \ldots \ldots \ldots \ldots \ldots \ldots \ldots$ & $0.0-1.0$ & $\ldots$ & 963 & $254(26.4 \%)$ & $\ldots$ & $\ldots$ & Spectroscopy \\
\hline
\end{tabular}

Notes.-In the notes below: HP spec.: high-precision exoplanet target spectroscopy; CdS spec.: Cayrel de Strobel et al. (2001) spectroscopy; uvby phot.: uvby

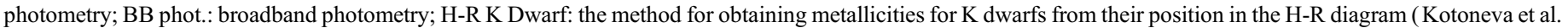
2002).

a Total number of Hipparcos Sun-like stars ( $H$ in Fig. 4).

b Subset of total stars that are hosts to stellar companions ( $S$ in Fig. 4). The percentages given correspond to the fraction $S / H$.

c Subset of total stars that are exoplanet target stars ( $T$ in Fig. 4). The percentages given correspond to the fraction $T / H$.

d Subset of target stars that are exoplanet hosts ( $P$ in Fig. 4). The percentages given correspond to the fraction $P / T$.

e $63 \% \mathrm{HP}$ spec., $12 \% \mathrm{CdS}$ spec., $20 \%$ uvby phot, $1 \% \mathrm{BB}$ phot, and $4 \% \mathrm{H}-\mathrm{R} \mathrm{K}$ dwarf.

f $19 \% \mathrm{HP}$ spec., $5 \% \mathrm{CdS}$ spec., $55 \%$ uvby phot, $17 \% \mathrm{BB}$ phot, and $4 \% \mathrm{H}-\mathrm{R} \mathrm{K}$ dwarf.

g $63 \%$ HP spec., $18 \%$ CdS spec., and 19\% uvby phot.

h $26 \%$ HP spec., $7 \%$ CdS spec., $61 \%$ uvby phot, $6 \%$ BB phot, and $<1 \%$ H-R K dwarf.

i $64 \%$ HP spec., $5 \%$ CdS spec., 20\% uvby phot, 3\% BB phot, and 8\% H-R K dwarf.

j $6 \%$ HP spec., $3 \%$ CdS spec., $44 \%$ uvby phot, $36 \%$ BB phot, and $11 \%$ H-R K dwarf.

${ }^{\mathrm{k}} \mathrm{GC}$ is the Geneva-Copenhagen survey of the solar neighborhood sample (Nordström et al. 2004). We only include those binaries observed by CORAVEL between 2 and 10 times (see $\S 4)$.

${ }^{1} \mathrm{CL}$ is the Carney-Latham survey of proper-motion stars (Carney et al. 2005). We include only those stars on prograde Galactic orbits $\left(V>-220 \mathrm{~km} \mathrm{~s}{ }^{-1}\right.$ ). The $\mathrm{CL}$ sample also includes 231 stars from the sample of Ryan (1989).

Almost all $(453 / 464=98 \%)$ of the close $(d<25$ pc $)$ sample and $2745 / 2832=97 \%$ of the more distant $(25 \mathrm{pc}<d<50 \mathrm{pc})$ sample thus have a value for metallicity. Given the different uncertainties associated with the five sources of metallicity, the close sample has more precise values of metallicity than the more distant sample. The dispersions for the close and far samples are 0.07 and 0.10 dex, respectively. See Table 1 for details.

We also investigate the color or host mass dependence of the host metallicity distributions. Thus, we split our close and far samples, which are defined by $0.5 \leq B-V \leq 1.0$, into 2 groups, those with $0.5 \leq B-V \leq 0.75$, which we call FG dwarfs, and those with $0.75<B-V \leq 1.0$, which we call $\mathrm{K}$ dwarfs. This split is shown in Table 1. In this table we also show the total number of stars in the sample that have a known value of metallicity and the fraction that are close binaries, exoplanet target stars, and exoplanet hosts.

In order to determine whether there is a real physical correlation between the presence of stellar or planetary companions and host metallicity, we need to show that there are only negligible selection effects associated with the detection and measurement of these two quantities that could cause a spurious correlation. In $\S 2.3$ we show that the planetary companion fraction should be complete for planetary companions with periods less than $5 \mathrm{yr}$ for the sample of target stars that are being monitored for exoplanets. This completeness helps assure a minimal spurious correlation between the probability of detecting planetary companions and host metallicity.

The stellar companion sample is made up of two subsamples: those companions detected as part of an exoplanet survey and those that were not. The target list for exoplanets is biased against stellar binarity, as discussed in Grether \& Lineweaver (2006). We show that there is negligible bias between the probability of detecting stellar companions and host metallicity in two ways: (1) by showing that our close sample of stellar companions is nearly complete and (2) by using the Geneva-Copenhagen (GC) survey of the solar neighborhood (Nordström et al. 2004) sample of stars, containing types of stars similar to those found in our sample, as an independent check on our results.

The GC sample of stars that contains F0-K3 stars is expected to be complete for stars with stellar companions closer than $d<$ $40 \mathrm{pc}$. For the close sample of stars $(d<25 \mathrm{pc})$, the northern hemisphere of stars with close stellar companions is approximately complete. The southern hemisphere of stars is also nearly complete, if we include the binary stars from Jones et al. (2002) that are likely to fall within our sample (Grether \& Lineweaver 2006). We then find that $\sim 10 \%$ of stars have stellar companions with periods shorter than 5 yr. If we make a small asymmetry correction (to account for the southern hemisphere not being as well monitored for binaries), we find that $\sim 11 \% \pm 3 \%$ of stars have stellar companions within this period range (Grether \& Lineweaver 2006). We also compare our sample with that of the Carney-Latham survey (CL) of proper-motion stars (Carney \& Latham 1987; Carney et al. 1994) in $\S 4$. The CL sample also contains $\sim 11 \%$ of stars with stellar companions with periods shorter than $5 \mathrm{yr}$ (Latham et al. 2002). We tabulate the properties of all these samples in Table 1.

\subsection{Close Companions}

The close companions included in our $d<25 \mathrm{pc}$ and $25 \mathrm{pc}<$ $d<50$ pc samples are enclosed in a rectangle of mass-period space shown in Figure 3. These companions have primarily been detected using the Doppler technique, but the stellar companions have been detected with a variety of techniques not exclusively 


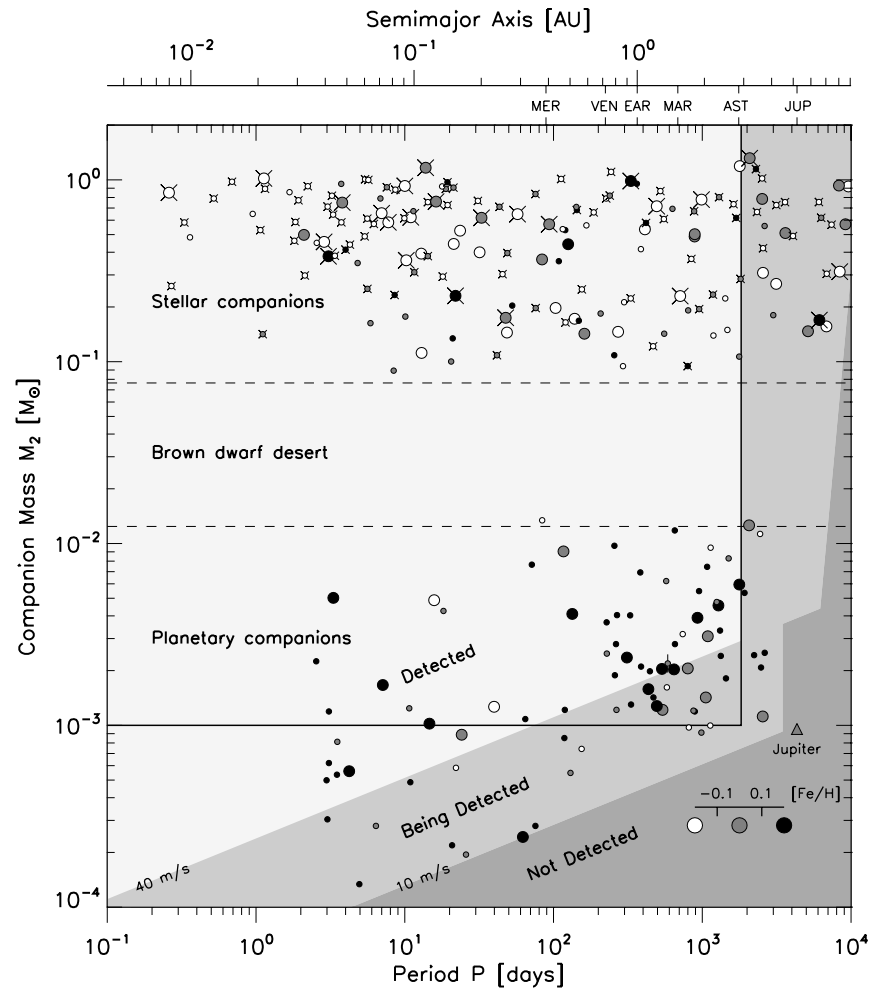

FIG. 3.-Masses and periods of close companions to stellar hosts of FGK spectral type. We split the close-companion sample into three groups defined by the metallicity of their host star: metal-poor $([\mathrm{Fe} / \mathrm{H}]<-0.1)$, Sun-like $(-0.1 \leq[\mathrm{Fe} / \mathrm{H}] \leq$ $0.1)$, and metal-rich $([\mathrm{Fe} / \mathrm{H}]>0.1)$, which are plotted as white, gray, and black dots, respectively. The larger points represent companions orbiting stars in the more complete $d<25 \mathrm{pc}$ sample, while the smaller points represent companions to stars at distances $d$ between 25 and 50 pc. We divide the stellar companions into those not monitored by one of the exoplanet search programs (shown with a cross behind the point) and those that are monitored. Both groups of stellar companions are distributed over the entire less biased region (enclosed by a thick line). Hence, any missing stellar companions should be randomly distributed. For multiple-companion systems, we select the most massive companion in our less biased sample to represent the system.

from high-precision exoplanet Doppler surveys. Thus, we need to consider the selection effects of the Doppler method in order to define a less biased sample of companions (Lineweaver \& Grether 2003). Given a fixed number of targets, the region labeled "Detected" should contain all companions that will be found for this region of mass-period space. The region labeled "Being Detected" should contain some but not all companions that will be found in this region, and the region labeled "Not Detected" contains no companions, since the current Doppler surveys are either not sensitive enough or have not been observing for a long enough duration to detect companions in this regime. Thus, as a consequence of the exoplanet surveys' limited monitoring duration and sensitivity for our sample, we select only those companions with an orbital period $P<5$ yr and mass $M_{2}>0.001 M_{\odot}$.

In Grether \& Lineweaver (2006) we found that companions with a minimum mass in the brown dwarf mass regime were likely to be low-mass stellar companions seen face-on, thus producing a very dry brown dwarf desert. We also included the 14 stellar companions from Jones et al. (2002) that have no published orbital solutions but are assumed to orbit with periods of $<5 \mathrm{yr}$. We find one new planet and no new stars in our less biased rectangle when compared with the data used in Grether \& Lineweaver (2006). This new planet, HD 20782 (HIP 15527), indicated by a vertical
TABLE 2

Metallicity and Frequency of Hosts with Close Planetary and Stellar Companions

\begin{tabular}{ccccrr}
\hline \hline Companions & Range & Total & Metal-poor & Sun-like & Metal-rich \\
\hline Planets .............. & $d<25$ & 19 & $2(11 \%)$ & $5(26 \%)$ & $12(63 \%)$ \\
Stars................. & $d<25$ & $45^{\mathrm{a}}$ & $25(56 \%)$ & $14(31 \%)$ & $6(13 \%)$ \\
Planets ............... & $25<d<50$ & 36 & $3(8 \%)$ & $9(25 \%)$ & $24(67 \%)$ \\
Stars................. & $25<d<50$ & $107^{\mathrm{b}}$ & $55(51 \%)$ & $36(34 \%)$ & $16(15 \%)$ \\
\hline
\end{tabular}

${ }^{\text {a }}$ An additional two hosts with unknown metallicity have stellar companions.

b An additional three hosts with unknown metallicity have stellar companions.

line through the point in Fig. 3), has been monitored for well over $5 \mathrm{yr}$, but has a period of only $\sim 1.6 \mathrm{yr}$ and a minimum mass of $1.8 M_{\text {Jup }}$, placing it just between the "Detected" and "Being Detected" regions. While most planets are detected within a time frame comparable to the period, the time needed to detect this planet was much longer than its period because of its unusually high eccentricity of 0.92 (Jones et al. 2006). We thus have two groups of close companions to analyze as a function of host metallicity, giant planets and stars.

In Figure 3 we split the close-companion sample into three groups defined by the metallicity of their host star: metal-poor $([\mathrm{Fe} / \mathrm{H}]<-0.1)$, Sun-like $(-0.1 \leq[\mathrm{Fe} / \mathrm{H}] \leq 0.1)$, and metalrich $([\mathrm{Fe} / \mathrm{H}]>0.1)$, which are plotted as white, gray, and black dots, respectively. Figure 3 suggests that the hosts of planetary companions are generally metal-rich, whereas the hosts of stellar companions are generally metal-poor. Table 2 and Figure 4 confirm the correlation between exoplanets and high metallicity and indicate an anticorrelation between stellar companions and high metallicity.

\section{CLOSE COMPANION-HOST METALLICITY CORRELATION}

We examine the distribution of close companions as a function of stellar host metallicity in our two samples. We do this quantitatively by fitting power-law and exponential best fits to the metallicity data expressed both linearly and logarithmically. We define the logarithmic $[\mathrm{Fe} / \mathrm{H}]$ and linear $Z / Z_{\odot}$ metallicity as follows:

$$
[\mathrm{Fe} / \mathrm{H}]=\log (\mathrm{Fe} / \mathrm{H})-\log (\mathrm{Fe} / \mathrm{H})_{\odot}=\log \left(\mathrm{Z} / \mathrm{Z}_{\odot}\right),
$$

where $\mathrm{Fe}$ and $\mathrm{H}$ are the number of iron and hydrogen atoms, respectively, and $Z=\mathrm{Fe} / \mathrm{H}$. We examine the close planetary companion probability $P_{\text {planet }}$ and the close stellar companion probability $P_{\text {star }}$ as a function of $[\mathrm{Fe} / \mathrm{H}]$ in Figures 4 and 6 for the $d<25$ and $25 \mathrm{pc}<d<50$ pc samples, respectively. Similarly, we also examine $P_{\text {planet }}$ and $P_{\text {star }}$ as a function of $Z / Z_{\odot}$ in Figures 5 and 7, which are effectively just a rebinning of the data in Figures 4 and 6, respectively. We then find the linear best fits to the planetary and stellar companion fraction distributions, as shown by the dashed lines in Figures 4-7.

We also fit an exponential to the $[\mathrm{Fe} / \mathrm{H}]$ planetary (as in Fischer $\&$ Valenti 2005) and stellar companion fraction distributions in Figures 4 and 6 (solid lines) and, equivalently, a power law to the data points for the $Z / Z_{\odot}$ plots, Figures 5 and 7 (solid lines). The two linear parameterizations that we fit to the data are

$$
\begin{aligned}
& P_{\text {lin } \mathrm{Fe} / \mathrm{H}}=a[\mathrm{Fe} / \mathrm{H}]+P_{\odot}, \\
& P_{\operatorname{lin} Z / Z_{0}}=A\left(Z / Z_{\odot}\right)+\left(P_{\odot}-A\right),
\end{aligned}
$$



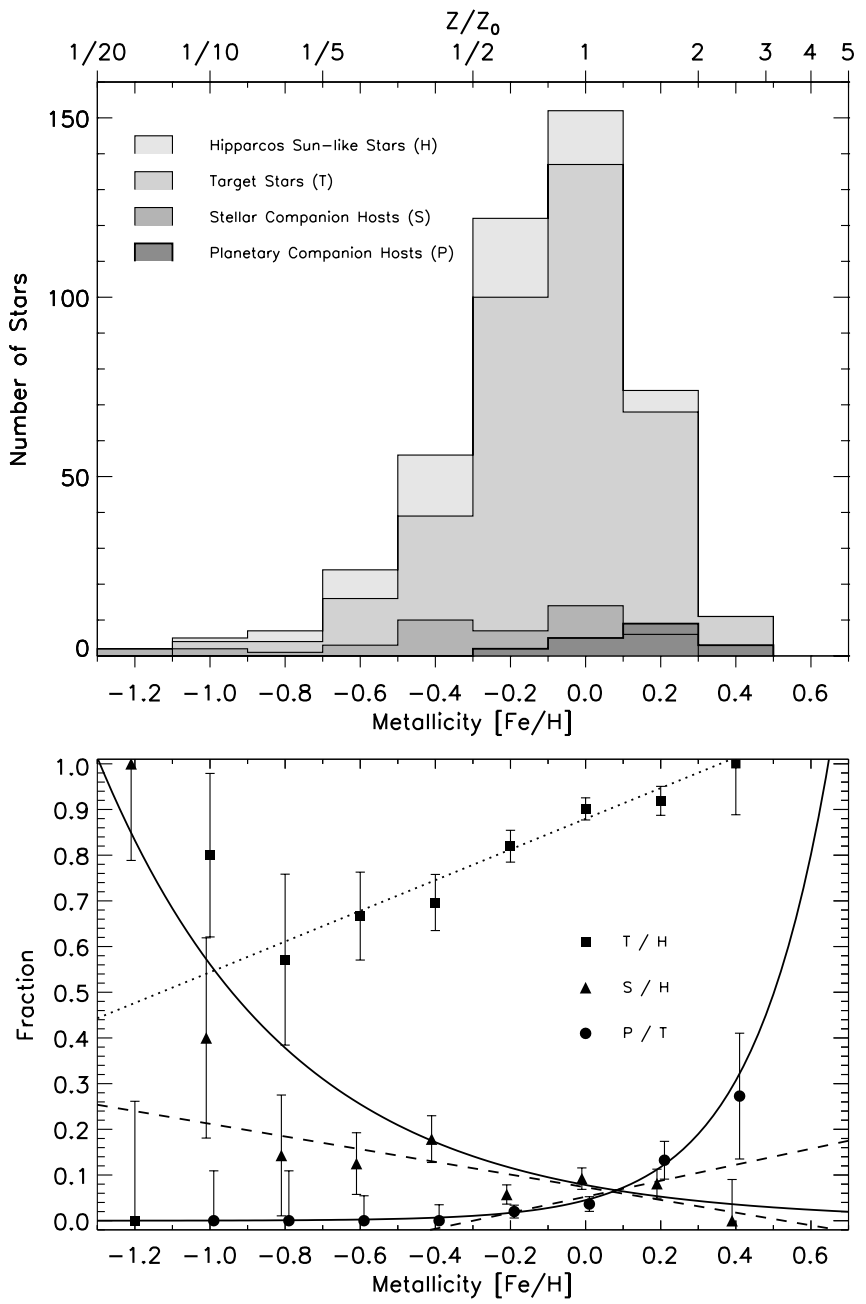

Fig. 4.-Metallicities $([\mathrm{Fe} / \mathrm{H}])$ of 453 stars in our close $d<25 \mathrm{pc}$ sample. Top: Hipparcos Sun-like stars in our close sample (lightest shade of gray), all of the stars that are exoplanet targets (next darker shade of gray), the hosts of stellar companions (still darker shade of gray), and the exoplanet hosts (darkest shade of gray). Bottom: Fraction of target stars (squares), stellar companion hosts (triangles), and planetary companion hosts (circles). The linear best fit to the target fraction is shown by a dotted line. The linear and exponential best fits to the stellar and planetary companion fractions are shown by dashed and solid lines, respectively.

and the two nonlinear parameterizations are

$$
\begin{aligned}
P_{\mathrm{EP}} & =P_{\odot} 10^{\alpha[\mathrm{Fe} / \mathrm{H}]} \\
& =P_{\odot}\left(Z / Z_{\odot}\right)^{\alpha},
\end{aligned}
$$

where $P_{\odot}$ is the fraction of stars of solar metallicity (i.e., $[\mathrm{Fe} / \mathrm{H}]=0$ and $\left.Z / Z_{\odot}=1\right)$ with companions.

If the fits for the parameters $a, A$, and $\alpha$ are consistent with zero, then there is no correlation between the fraction of stars with companions and metallicity. On the other hand, a nonzero value, several $\sigma$ away from zero, suggests a significant correlation ( $a, A$, or $\alpha>0)$ or anticorrelation $(a, A$, or $\alpha<0)$.

The best-fit parameters $a, A$, and $P_{\odot}$ (but not $\alpha$ ) depend on the period range and completeness of the sample. In order to compare the slopes from different samples, we parameterize this dependence in terms of the average companion fraction $P_{\text {avg }}$ for the sample; i.e., if the average companion fraction for a sample is twice as large as for another sample, the best-fit slopes $a$ and $A$, as well as the fraction of stars of solar metallicity, $P_{\odot}$, will also be twice as large. To compare samples with different $P_{\text {avg }}$, we scale
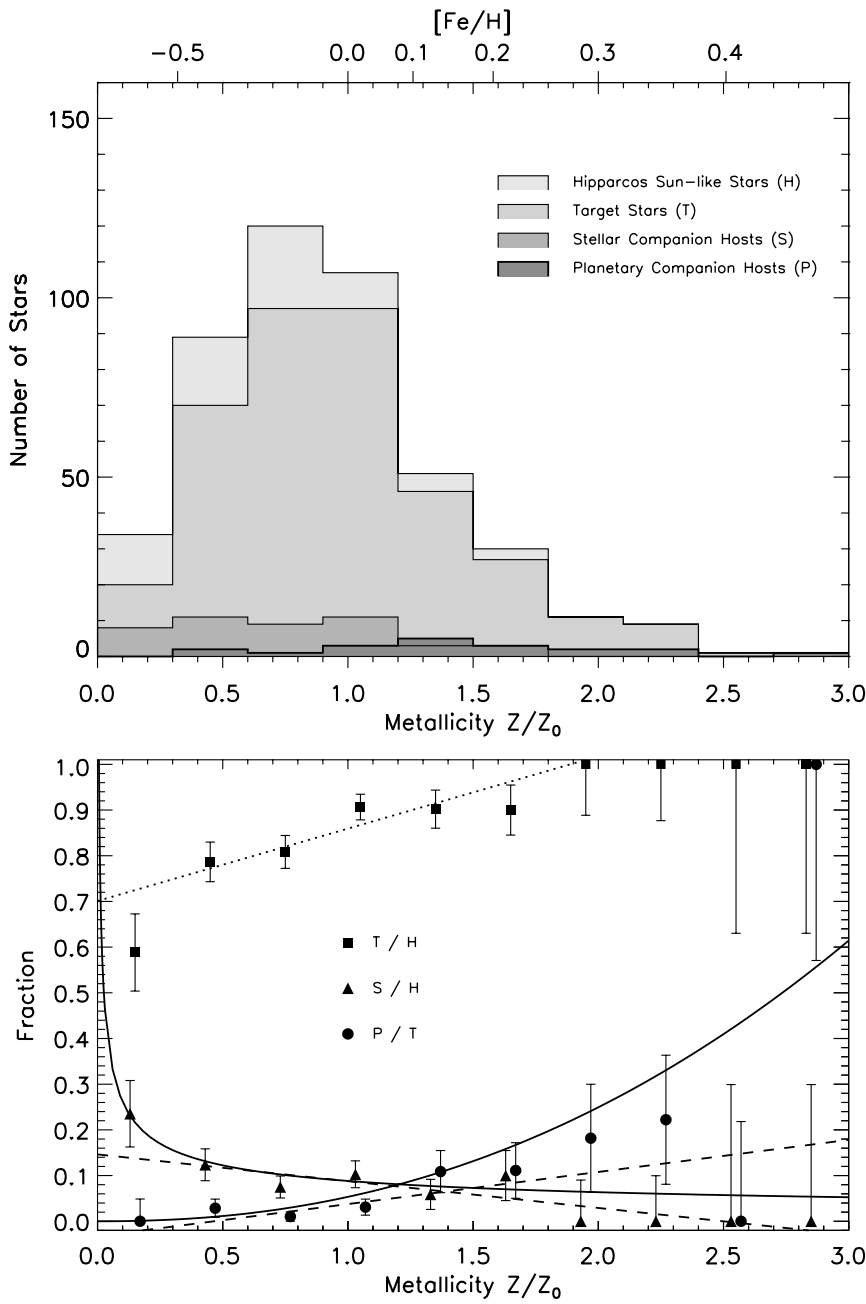

FIG. 5.-Same as Fig. 4, except that metallicity is plotted linearly as $Z / Z_{\odot}$. All of the metal-rich $\left(Z / Z_{\odot}>1.8\right)$ sample stars are being monitored for exoplanets, but as the stellar metallicity decreases, so does the fraction being monitored. This is because of a bias toward selecting more metal-rich target stars for observation due to an increased probability of planetary companions orbiting metal-rich host stars. The linear best fit to the target fraction is shown by a dotted line. The linear and power-law best fits to the stellar and planetary companion fractions are shown by dashed and solid lines, respectively.

the best-fit equations (2)-(5) to a common average companion fraction by dividing each equation by $P_{\text {avg. }}$. Thus, we scale the bestfit parameters $a, A$, and $P_{\odot}$ by dividing each by $P_{\text {avg. }}$. These scaled parameters are then referred to as $a^{\prime}=a / P_{\mathrm{avg}}, A^{\prime}=A / P_{\mathrm{avg}}$, and $P_{\odot}^{\prime}=P_{\odot} / P_{\text {avg }}$. We list the unscaled best-fit parameters $a, A, P_{\odot}$, and $\alpha$, along with $P_{\text {avg }}$, for each sample in Table 3 . The parameters $a^{\prime}=a / P_{\text {avg }}$ and $\alpha$ of the different samples are compared in Figure 11.

We consistently find in Figures 4 and 6 that metal-rich stars are being monitored more extensively for exoplanets than metal-poor stars, as quantified by the (target stars)/(Hipparcos Sun-like stars) $T / H$ ratio. This is because of a bias toward selecting more metalrich stars for observation due to an increased probability of planetary companions orbiting metal-rich host stars. Note that this bias is well represented by a linear trend, as shown by the dotted best-fit line in these figures, and is not just a case of a few high-metallicity stars being added to the highest metallicity bins. We correct for this bias by calculating $\mathrm{P} / \mathrm{T}$, not $\mathrm{P} / \mathrm{H}$, for each metallicity bin.

We find a correlation between $[\mathrm{Fe} / \mathrm{H}]$ and the presence of planetary companions in Figure 4 . The linear best fit (eq. [2]) has a gradient of $a=0.18 \pm 0.07\left(\chi_{\text {red }}^{2}=1.21\right)$, and thus the correlation 


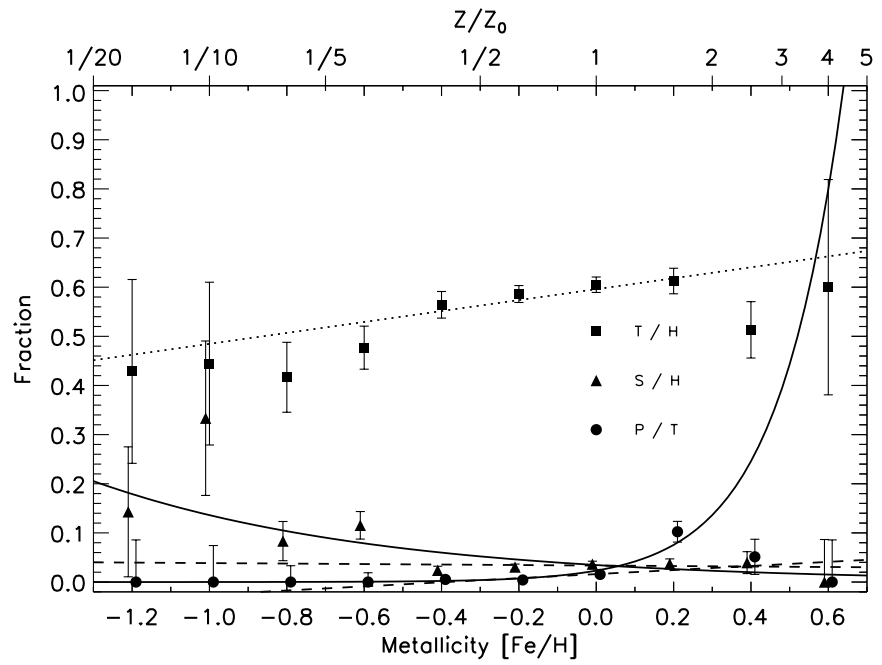

FIG. 6.- Same as Fig. 4, except for the 2745 stars in the more distant 25 pc $<$ $d<50 \mathrm{pc}$ sample. It is harder to detect distant planets because of signal-to-noise ratio considerations, which limit observations to the brighter stars. This fainter, more distant sample relies more on photometric metallicity determinations than does the closer, brighter sample, which has predominantly spectroscopic metallicity determinations (see Table 1). The fraction of stars being monitored for exoplanets is much lower than in Fig. 4.

is significant at the $2 \sigma$ level. The nonlinear best fit (eq. [4]) is $\alpha=2.09 \pm 0.54\left(\chi_{\text {red }}^{2}=0.16\right)$, and thus the correlation is significant at slightly more than the $3 \sigma$ level.

Similarly, we find a correlation between linear metallicity $Z / Z_{\odot}$ and the presence of planetary companions in the same data rebinned in Figure 5. The linear best fit (eq. [3]) has a gradient of $A=0.07 \pm 0.03\left(\chi_{\text {red }}^{2}=1.25\right)$, and the nonlinear best fit (eq. [5]) has an exponent of $\alpha=2.22 \pm 0.39\left(\chi_{\text {red }}^{2}=1.00\right)$, which are nonzero at the $\sim 2$ and $\sim 5 \sigma$ significance levels, respectively. These results are summarized in Table 3 . We can compare the nonlinear best fit (eq. [5]) for linear metallicity $Z / Z_{\odot}$ and the nonlinear best fit (eq. [4]) for $\log$ metallicity [Fe/H], since both contain the parameter $\alpha$. As shown by the $\chi^{2}$ per degree of freedom, $\chi_{\text {red }}^{2}$, the nonlinear goodness of fit is better than the linear goodness of fit. We rely on the best-fitting functional form that is the nonlinear parameterization of our results, although we use both parameterizations in our analysis.

We combine these two nonindependent, nonlinear best-fit estimates by computing their weighted average. We assign an error to this average by adding in quadrature (1) the difference between the two estimates and (2) the nominal error on the average. Thus, our best estimate is $\alpha=2.2 \pm 0.5$. Hence, the correlation between the presence of planetary companions and host metallicity is significant at the $\sim 4 \sigma$ level for a nonlinear best fit and at the $\sim 2 \sigma$ level with a lower goodness of fit for a linear best fit in our close, most complete sample.

In Figure 4 and its rebinned equivalent, Figure 5, we find an anticorrelation between the presence of stellar companions and host metallicity. The linear stellar companion best fits have gradients of $a=-0.14 \pm 0.06\left(\chi_{\text {red }}^{2}=3.00\right)$ and $A=-0.06 \pm$ $0.03\left(\chi_{\text {red }}^{2}=0.91\right)$, respectively, both significant at the $\sim 2 \sigma$ level. The nonlinear best fit to the stellar companions as a function of $[\mathrm{Fe} / \mathrm{H}]$ in Figure 4 is $\alpha=-0.86 \pm 0.10\left(\chi_{\text {red }}^{2}=1.33\right)$, and the nonlinear best fit to the stellar companions as a function of $Z / Z_{\odot}$ in Figure 5 is $\alpha=-0.47 \pm 0.18\left(\chi_{\text {red }}^{2}=0.40\right)$. Averaging these two as above, we obtain $-0.8 \pm 0.4$, which is significant at the $\sim 2 \sigma$ level. All these best fits are summarized in Table 3 .

Having found a correlation for planetary companions and an anticorrelation for stellar companions in our close sample and

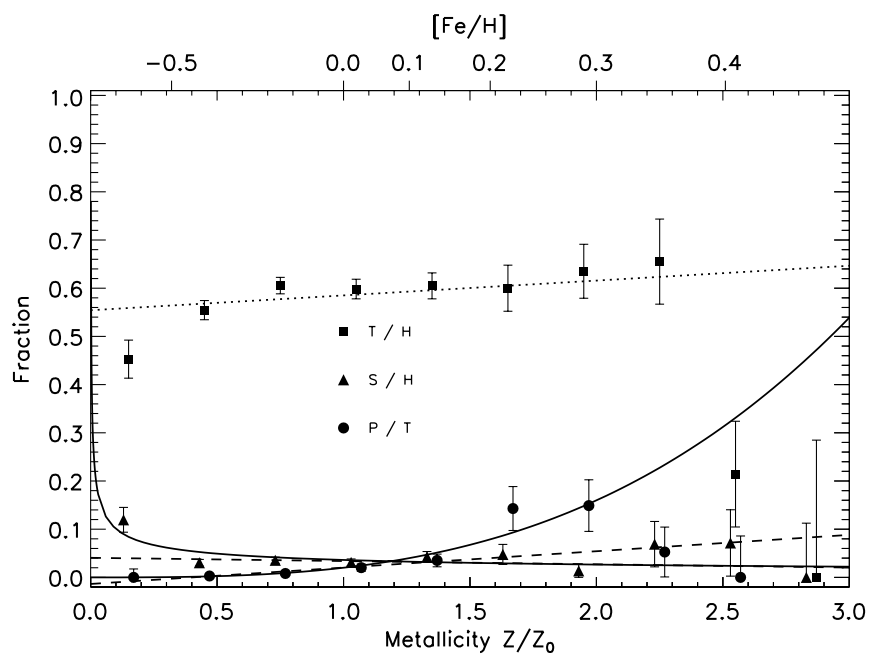

FIG. 7.- Same as Fig. 6, except that metallicity is plotted linearly as $Z / Z_{\odot}$, analogous to Fig. 5. In this more distant sample, we find the same trends as in Fig. 5, but they are not as prominent.

having found them to be robust to different metallicity binnings, we perform various other checks to confirm their reality. We check the robustness of both results to (1) distance and (2) spectral type ( $\sim$ mass) of the host star.

To check whether these anticorrelations have a distance dependence, we repeat this analysis for the less complete $25 \mathrm{pc}<$ $d<50$ pc sample. As shown by the best fits in Figures 6 and 7 and summarized in Table 3, we find only a marginal anticorrelation between the presence of stellar companions and host metallicity for the linear best fits. The nonlinear best fits, however, still suggest an anticorrelation with $\alpha=-0.59 \pm 0.12$ for $\log$ metallicity $[\mathrm{Fe} / \mathrm{H}]$ and $\alpha=-0.44 \pm 0.12$ for linear metallicity $Z / Z_{\odot}$, which are significant at the 4 and $3 \sigma$ levels, respectively. Combining these two estimates as described above, we find $\alpha=$ $-0.5 \pm 0.2$, significant at the $2 \sigma$ level in the $25 \mathrm{pc}<d<50 \mathrm{pc}$ sample.

The correlation between the presence of planetary companions and host metallicity for the less complete $25 \mathrm{pc}<d<50 \mathrm{pc}$ is significant at the 4 and $3 \sigma$ levels for the linear best fits $a$ and $A$, respectively. The nonlinear best-fit correlation has $\alpha=2.56 \pm$ 0.45 for $\log$ metallicity $[\mathrm{Fe} / \mathrm{H}]$ and $\alpha=3.00 \pm 0.46$ for linear metallicity $Z / Z_{\odot}$, which are significant at the 5 and $6 \sigma$ levels, respectively. Combining these two estimates, we find the weighted average as above of $\alpha=2.8 \pm 0.6$, significant at the $4 \sigma$ level.

Having found the correlation for planetary companions and the anticorrelation for stellar companions robust to binning but less robust in the less complete, more distant sample, we test for spectral type ( host mass) dependence. We split our sample into bluer and redder subsamples to investigate the effect of spectral type on the close companion-host metallicity relationship. We define the bluer subsample as $B-V \leq 0.75$ (FG spectral type stars) and the redder subsample as $B-V>0.75$ (K spectral type stars). Since $B-V$ has a metallicity dependence, a cut in $B-V$ will not be a true mass cut, but a diagonal cut in mass versus metallicity. Thus, interpreting a $B-V$ cut as a pure cut in mass introduces a spurious anticorrelation between mass and metallicity.

The linear best fit to the stellar companions of the FG sample $(d<25 \mathrm{pc})$ has a normalized gradient of $a^{\prime}=(-0.01 \pm$ $0.08) / 10.5 \%=-0.1 \pm 0.8$, and the nonlinear best fit is $\alpha=$ $-0.2 \pm 0.4$, as shown in Figure 8. Both of these best fits are consistent with the frequency of stellar companions being independent of host metallicity. The linear best fit to the stellar companions of 
TABLE 3

Best-Fit Trends for Close Companion-Host Metallicity Correlation

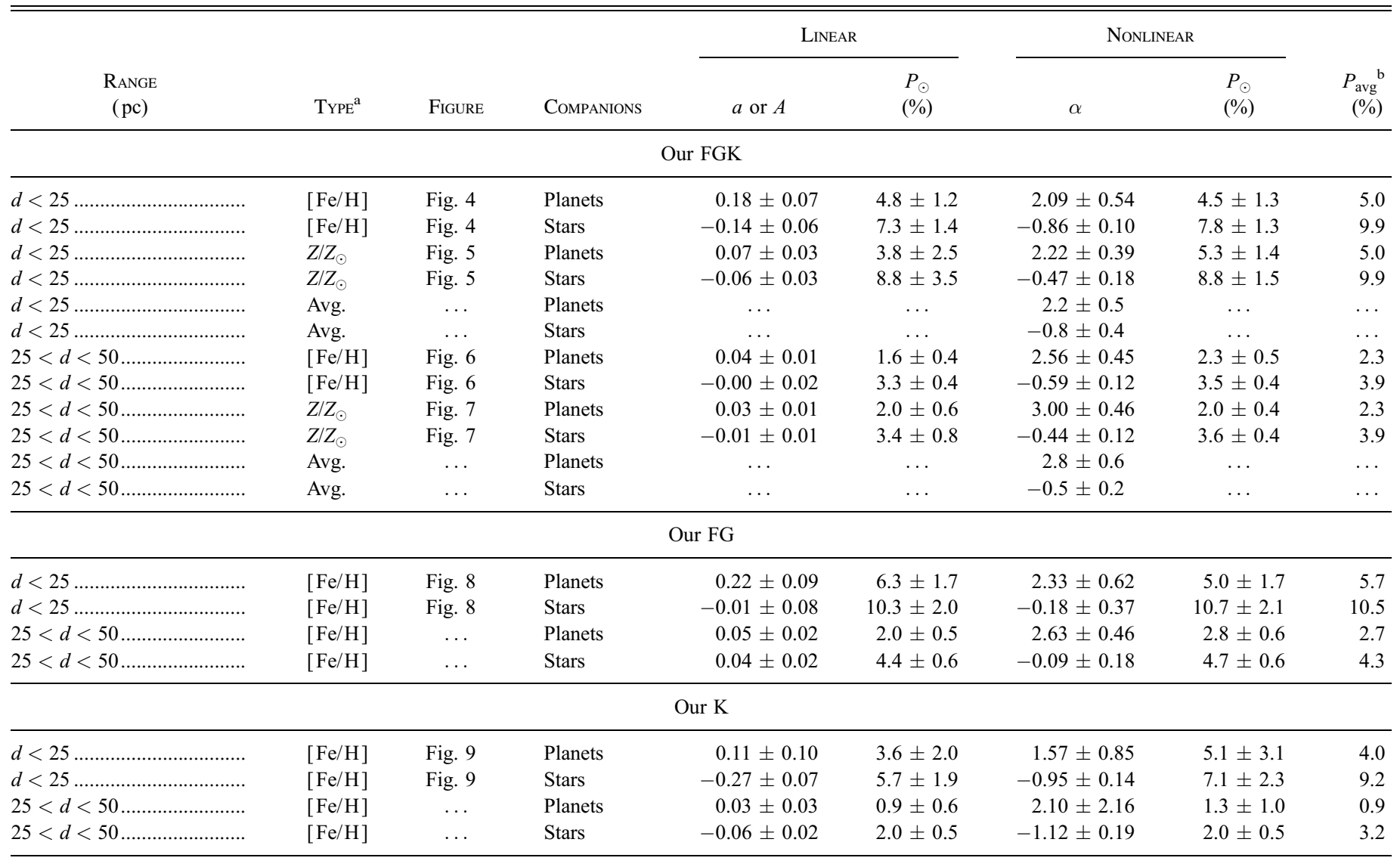

$\mathrm{GC}^{\mathrm{c}} \mathrm{FGK}$

\begin{tabular}{|c|c|c|c|c|c|c|c|c|}
\hline$d<40$ & {$[\mathrm{Fe} / \mathrm{H}]$} & Fig. 12 & Stars & $-0.15 \pm 0.05$ & $24.0 \pm 1.5$ & $-0.28 \pm 0.07$ & $23.9 \pm 1.4$ & 26.8 \\
\hline \multicolumn{9}{|c|}{ GC FG } \\
\hline \multicolumn{9}{|c|}{ GC K } \\
\hline$d<40$ & {$[\mathrm{Fe} / \mathrm{H}]$} & Fig. 14 & Stars & $-0.46 \pm 0.10$ & $22.3 \pm 3.6$ & $-0.52 \pm 0.11$ & $23.7 \pm 3.9$ & 32.0 \\
\hline & {$[\mathrm{Fe} / \mathrm{H}]$} & $\ldots$ & Stars & $-0.07 \pm 0.06$ & $21.3 \pm 3.4$ & $-0.12 \pm 0.09$ & $21.6 \pm 3.1$ & 25.7 \\
\hline \multicolumn{9}{|c|}{ Combined $^{\mathrm{e}}$} \\
\hline & {$[\mathrm{Fe} / \mathrm{H}]$} & Fig. 15 & Stars & $-0.10 \pm 0.03$ & $20.5 \pm 1.3$ & $-0.22 \pm 0.05$ & $20.7 \pm 1.2$ & 25.7 \\
\hline
\end{tabular}

Note.-See Fig. 11.

a "Type" refers to whether the data are binned in $[\mathrm{Fe} / \mathrm{H}]$ or in $Z / Z_{\odot}$. For data binned in $[\mathrm{Fe} / \mathrm{H}]$, the linear slope is $a$, and for data binned in $Z / Z_{\odot}$, the linear slope is $A$ (see eqs. [2]-[5]).

${ }^{b} P_{\text {avg }}$ is defined as the number of stars with stellar companions divided by the total number of stars. We use this parameter to scale $a, A$, and $P_{\odot}$, which are then referred to as $a^{\prime}=a / P_{\text {avg }}, A^{\prime}=A / P_{\text {avg }}$, and $P_{\odot}^{\prime}=P_{\odot} / P_{\text {avg }}$ and can be compared for different samples (see text, $\S 3$ ).

${ }^{c} \mathrm{GC}$ is the Geneva-Copenhagen survey of the solar neighborhood sample (Nordström et al. 2004). We include only those binaries observed by CORAVEL between 2 and 10 times (see $\S 4$ ).

${ }^{\mathrm{d}} \mathrm{CL}$ is the Carney-Latham survey of proper-motion stars (Carney et al. 2005). We include only those stars on prograde Galactic orbits $\left(V>-220 \mathrm{~km} \mathrm{~s}^{-1}\right)$ with $[\mathrm{Fe} / \mathrm{H}]>-1.3$. This also includes stars from the sample of Ryan (1989).

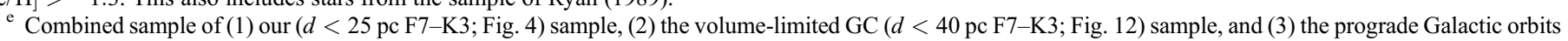
from Carney et al. (2005). Fig. 15 shows all three data sets. 

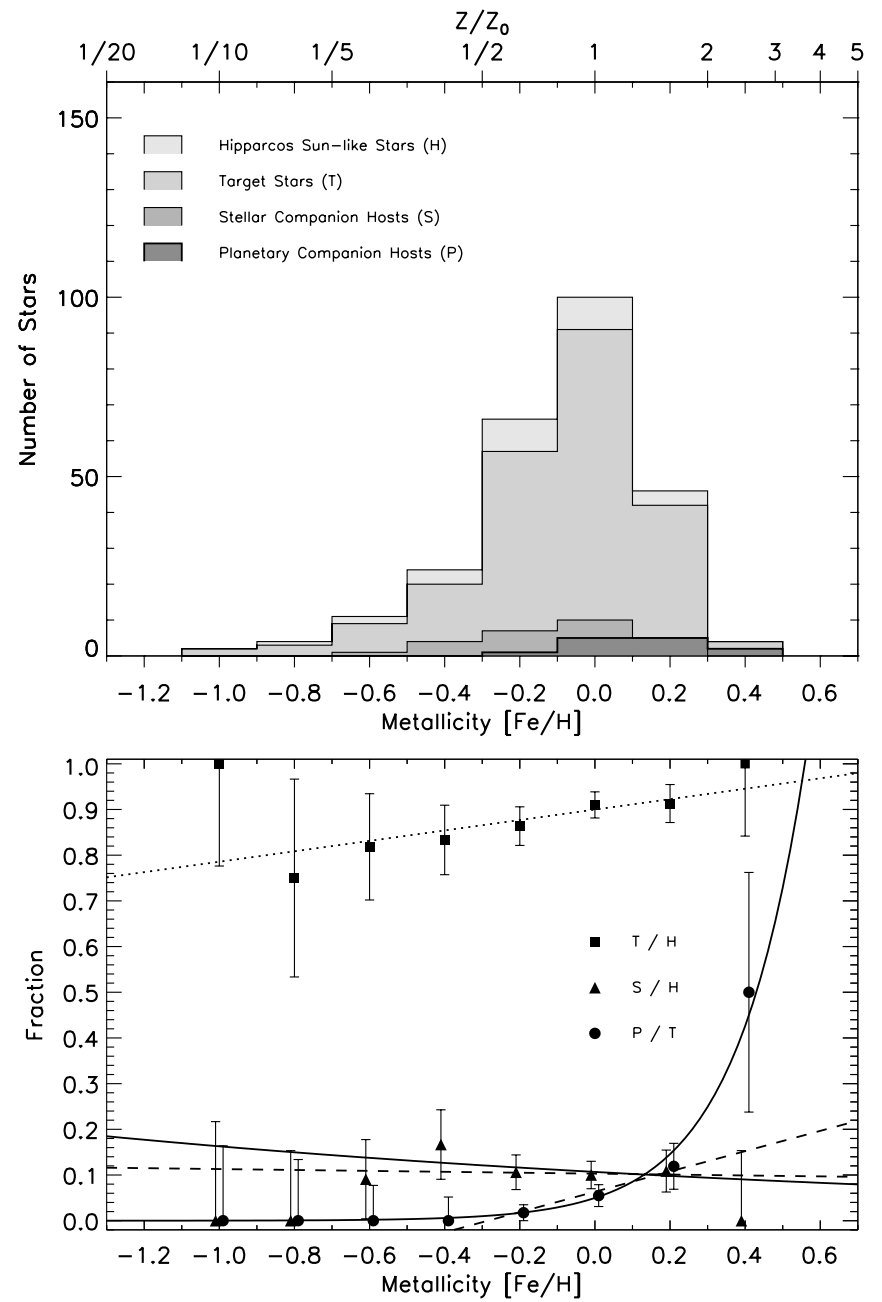

Fig. 8.-Same as Fig. 4 for the stars in our close $d<25$ pc sample, but only for FG dwarfs $(B-V \leq 0.75)$. All stars have known metallicity in this sample. There is no apparent anticorrelation between metallicity and the presence of stellar companions.

the $\mathrm{K}$ sample has a gradient of $a^{\prime}=(-0.27 \pm 0.07) / 9.2 \%=$ $2.9 \pm 0.8$, and the nonlinear best fit is $\alpha=-1.0 \pm 0.1$, as shown in Figure 9 for the close $d<25 \mathrm{pc}$ stars. Both of these best fits show an anticorrelation between the presence of stellar companions and host metallicity above the $3 \sigma$ level. Less significant results are obtained for the $25<d<50 \mathrm{FG}$ and $\mathrm{K}$ spectral type samples with stellar companions, as shown in Table 3. These results suggest that the observed anticorrelation between close binarity and host metallicity is either (1) real and stronger for $\mathrm{K}$ spectral type stars than for FG stars or (2) due to a spectral typedependent selection effect.

Under the hypothesis that the anticorrelation between host metallicity and binarity is real for $\mathrm{K}$ dwarfs, there is a possible selection effect limited to $\mathrm{F}$ and $\mathrm{G}$ stars that could explain why we do not see the anticorrelation as strongly in them. Doppler broadening of the line profile, due to both random thermal motion in the stellar atmosphere and stellar rotation, increases in more massive $\mathrm{F}$ and $\mathrm{G}$ stars due to their higher effective temperature and faster rotation speeds compared with less massive $\mathrm{K}$ stars. This wider line profile for $\mathrm{F}$ and $\mathrm{G}$ stars results in fewer observable shifting lines, thus lowering the spectroscopic binary detection efficiency. However, we directly examine the stellar companion fraction as a function of spectral type or color $B-V$ in Figure 10. For both single-lined and double-lined spectroscopic binaries, if the binary
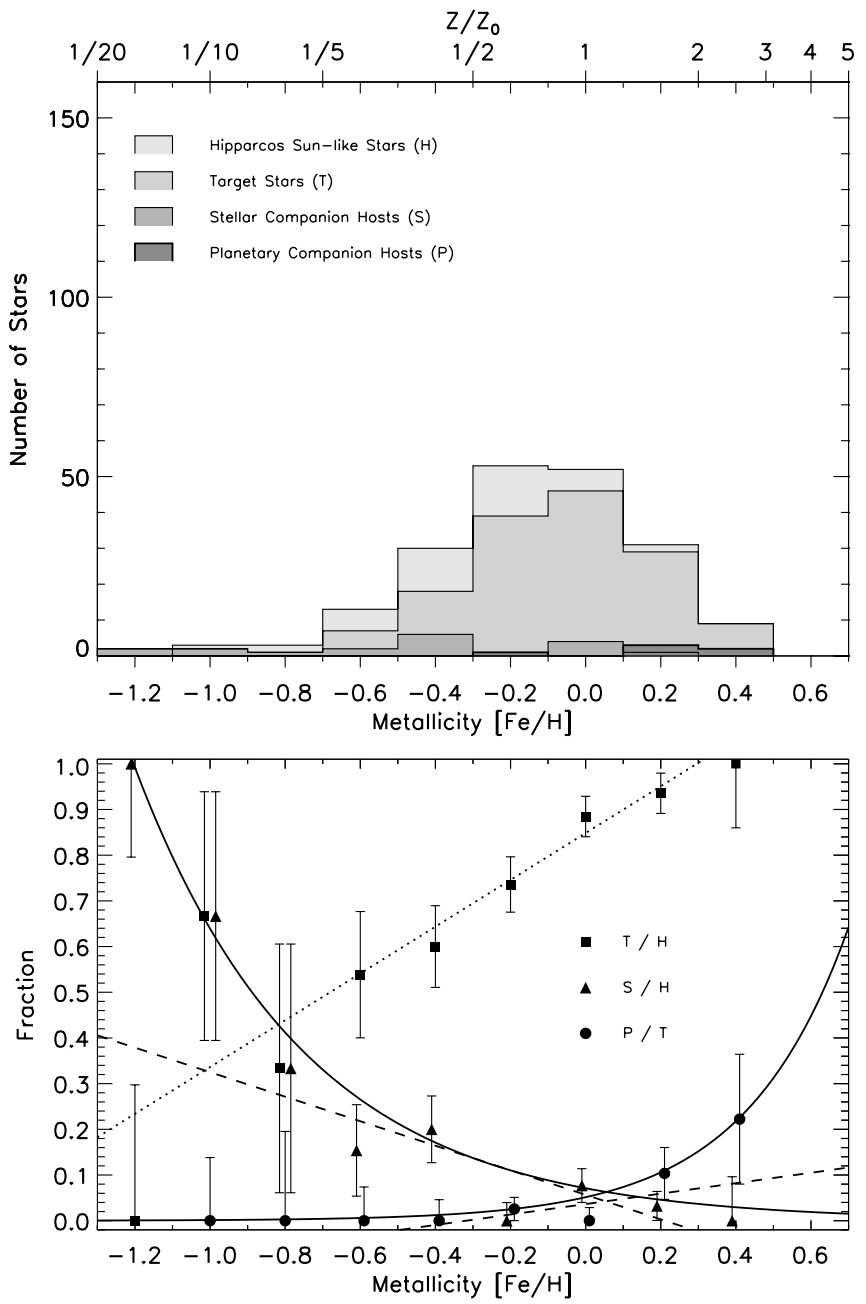

FIG. 9.- Same as Fig. 4 for the stars in our close $d<25$ pc sample, but only for $\mathrm{K}$ dwarfs $(B-V>0.75)$. This plot shows a strong anticorrelation between metallicity and the presence of stellar companions.

detection efficiency was systemically higher for K dwarfs, then the anticorrelation could be a selection effect. However, we find that it is fairly independent of spectral type. Thus, the anticorrelation does not appear to be a spectral type-dependent selection effect.

We also examine the spectral type ( $\sim$ mass) dependence of the correlation between planetary companions and host metallicity. The linear best fit to the planetary companions of the FG sample has a gradient of $a^{\prime}=(0.22 \pm 0.09) / 5.7 \%=3.9 \pm 1.6$, and the nonlinear best fit is $\alpha=2.3 \pm 0.6$, as shown in Figure 8 for the close $d<25 \mathrm{pc}$ stars. These are significant at the 2 and $3 \sigma$ levels, respectively. The linear best fit to the planetary companions of the K sample has a gradient of $a^{\prime}=(0.11 \pm 0.10) / 4.0 \%=$ $2.8 \pm 2.5$, and the nonlinear best fit is $\alpha=1.6 \pm 0.9$, as shown in Figure 9 for the close $d<25$ pc stars. These are both significant at levels between 1 and $2 \sigma$. The $\mathrm{K}$ sample contains fewer planetary and stellar companions compared to the FG sample. Both the linear and nonlinear fits are consistent for the FG and $\mathrm{K}$ samples, suggesting that the correlation between the presence of planetary companions and host metallicity is independent of spectral type and, consequently, host mass. The fraction of planetary companions is also fairly independent of spectral type, as shown in Figure 10.

Thus, our results suggest that the correlation between the presence of planetary companions and host metallicity is significant at the $\sim 4 \sigma$ level and that the anticorrelation between the presence 


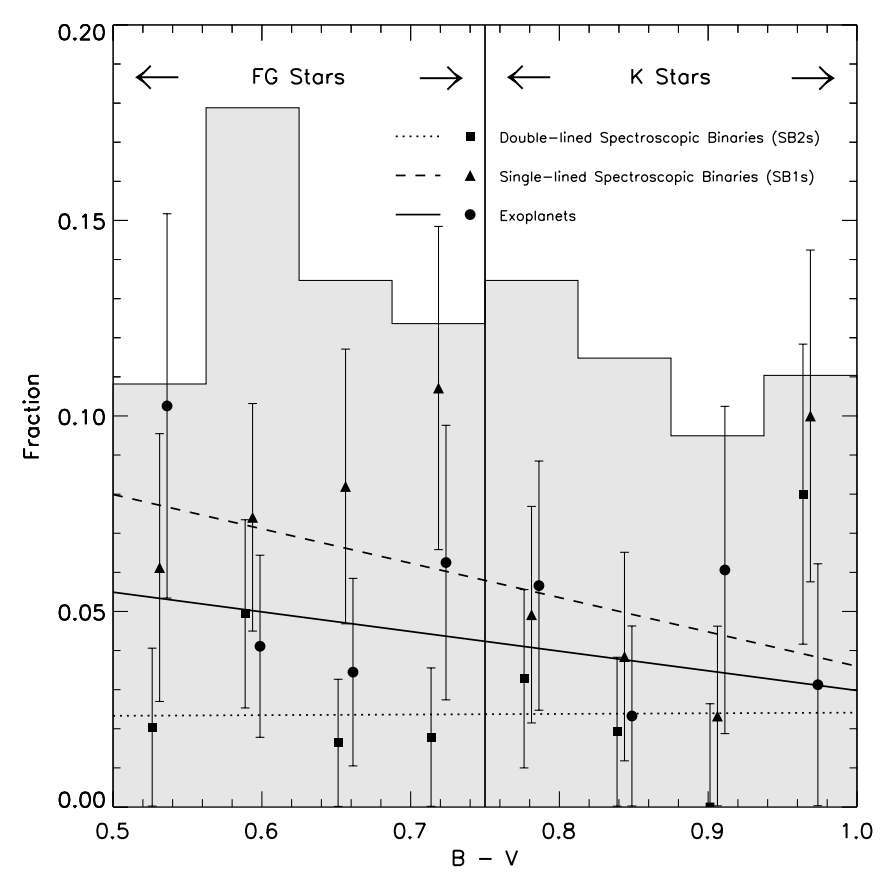

FIG. 10.-Color $(B-V)$ distribution for double-lined (squares) and singlelined (triangles) spectroscopic binaries (SB2s and SB1s, respectively) and exoplanets (circles) in our close $d<25$ pc sample. The linear best-fit gradient for SB2s is $0.00 \pm 0.06$, for SB1s it is $-0.08 \pm 0.08$, and for exoplanets it is $-0.05 \pm 0.08$. All three of these gradients are significant only at the $\lesssim 1 \sigma$ level. There is no significant correlation between SB1, SB2, or planetary fraction for either FG $(B-$ $V \leq 0.75)$ stars or $\mathrm{K}(B-V>0.75)$ stars.

of stellar companions and host metallicity is significant at the $\sim 2 \sigma$ for the $d<25$ pc FGK sample. Splitting both samples into $\mathrm{FG}$ and $\mathrm{K}$ spectral type stars suggests that the correlation between the presence of planetary companions and host metallicity is independent of spectral type, but that the anticorrelation between the presence of stellar companions and host metallicity is a strong function of spectral type, with the anticorrelation disappearing for the bluer FG host stars (see Fig. 11). We find no spectral type-dependent binary detection efficiency bias that can explain this anticorrelation.

\section{IS THE ANTICORRELATION BETWEEN METALLICITY AND STELLAR BINARITY REAL?}

We further examine the relationship between stellar metallicity and binarity by comparing our sample with that of the Geneva and Copenhagen survey (GC) of the solar neighborhood (Nordström et al. 2004), which has been selected as a magnitude-limited sample, a volume-limited portion $(d<40 \mathrm{pc})$ of which we analyze. These selection criteria imply that the sample is kinematically unbiased; i.e., the sample contains the same proportion of thin, thick, and halo stars as found in the solar neighborhood. We also compare our sample with that of the Carney-Latham survey (CL) that has been kinematically selected to have high proper motion stars (Carney \& Latham 1987); i.e., it contains a larger proportion of halo stars compared to disk stars than is observed for the solar neighborhood.

Our sample is based on the Hipparcos sample that has a limiting magnitude for completeness of $V=7.9+1.1 \sin |b|$ (Reid 2002), where $b$ is Galactic latitude. Thus, the Hipparcos sample is more complete for stars at higher Galactic latitudes, where the proportion of halo stars to disk stars increases. Hence our more distant $(25 \mathrm{pc}<d<50 \mathrm{pc})$ sample will have a small kinematic bias in that it will have an excess of halo stars, whereas our closer $(d<25$ pc) sample will be less kinematically biased.

\subsection{Comparison with a Kinematically Unbiased Sample}

The GC sample contains primarily F and G dwarfs with apparent visual magnitudes $V \lesssim 9$ and is complete in volume for $d<40$ pc for F0-K3 spectral type stars. Unlike our sample analyzed in $\S 3$, it also includes early $F$ spectral type stars. The GC sample color range is defined in terms of $b-y$, not $B-V$ as for our samples. We remove these early $\mathrm{F}$ stars with $b-y<0.3$ ( $B-V \lesssim 0.5$; Cox 2000) from the sample so that the GC sample spectral type range is similar to ours. The GC sample then ranges from $0.3 \leq b-y \leq 0.6(0.5 \lesssim B-V \lesssim 1.0)$, with those stars above $b-y=0.5(B-V \sim 0.75)$ referred to as K stars. We also exclude suspected giants from the GC sample.

For the GC sample, we include only those binaries observed by CORAVEL between 2 and 10 times so as to avoid a potential bias in which low-metallicity stars were observed more often, thus leading to a higher efficiency for finding binaries around these stars. This homogenizes the binary detection efficiency such that any real signal will not be removed by such a procedure. Unlike our sample, for which we include only binaries with $P<5 \mathrm{yr}$, the GC sample also includes much longer period visual binaries, in addition to short-period spectroscopic binaries, such that the total binary fraction of all types corresponds to $\sim 25 \%$. Comparing this with the period distribution for G dwarf stars of Duquennoy \& Mayor (1991), this binary fraction corresponds to binary systems with periods less than $\sim 10^{5}$ days.

For the volume-limited $d<40$ pc sample, we again find an anticorrelation between binarity and stellar host metallicity, as shown in Figure 12. Both the linear and nonlinear best fits listed in Table 3 are significant at or above the $3 \sigma$ level. We also split the GC sample into FG and K spectral type stars in Figures 13 and 14, respectively. The anticorrelation between the presence of stellar companions and host metallicity is significant at less than the $1 \sigma$ level for FG stars, but significant at the $\sim 4 \sigma$ level for $\mathrm{K}$ stars.

These results are qualitatively the same as those found for our sample, but quantitatively weaker, as shown in Figure 11 (see rows of points labeled GC). This may be due to the higher fraction of late $\mathrm{F}$ and early $\mathrm{G}$ spectral type stars compared to our samples or the larger range ( $\sim 10^{5}$ days) in binary periods contained in the GC sample compared to our sample, where $P<5$ yr. Another way of interpreting this anticorrelation between binarity and metallicity may be in terms of the age and nature of different components of the Galaxy described by stellar kinematics; i.e., F stars are generally younger than $\mathrm{K}$ stars and thus are more likely to belong to the younger thin-disk star population than the older thickdisk star population. Hence, we examine our results in terms of stellar kinematics.

\subsection{Comparison with a Kinematically Biased Sample}

We also compare our samples and that of the GC survey with the Carney \& Latham (1987) high proper motion survey (CL). The CL survey contains all of the A, F, and early G, many of the late $\mathrm{G}$, and some of the early $\mathrm{K}$ dwarfs from the Lowell Proper Motion Catalog (Giclas et al. 1971, 1978) that were also contained in the NLTT Catalog (Luyten 1979, 1980). The number of stars in this distribution increases as the stellar colors become redder, peaking at about $B-V=0.65$, following which the numbers of stars begin to decrease (Carney et al. 1994). This group has also obtained data for a smaller number of stars from the sample of Ryan (1989), who sampled subdwarfs (metal-poor stars beneath the main sequence) that have a high fraction of halo stars in the 


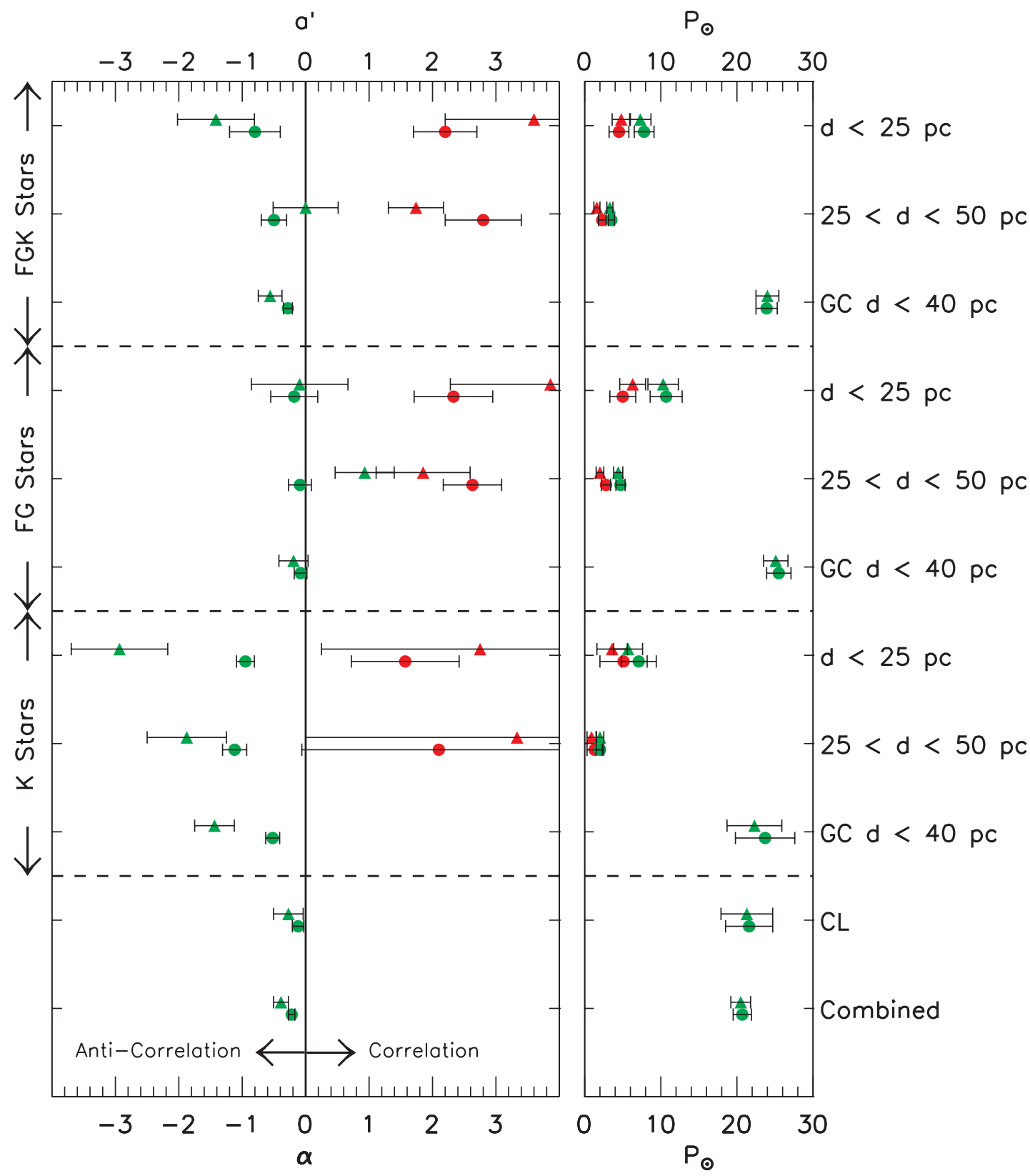

FIG. 11.-We compare the linear $a^{\prime}$ (triangles) and nonlinear $\alpha$ (circles) parameterizations for the various samples listed in Table 3. The red points are the best fits to planetary companions, and the green points are the best fits to stellar companions. The fact that the red planet values for $\alpha$ are significantly larger than zero confirms and quantifies the metallicity-planet correlation. The fact that the green stellar values for $\alpha$ are predominantly less than zero, significantly so only for $\mathrm{K}$ dwarfs, is a surprising new result. The labels on the right-hand side refer to the samples for which the best-fit parameterizations are valid. We normalize the linear parameterization by dividing the best-fit gradient $a$ by the average companion fraction $P_{\text {avg }}$ (see text). This plot is a graphical version of Table 3 , whose notes also apply to this plot. All of the best fits are from $[\mathrm{Fe} / \mathrm{H}]$ plots except for our FGK stars, where $\alpha$ is the average of the best fits to both the $[\mathrm{Fe} / \mathrm{H}]$ and $Z / Z_{\odot}$ plots. The $P_{\odot}$ values plotted in the vertical panel on the right refer to the corresponding best-fit normalization at solar metallicity (eqs. [2]-[5])

range $0.35<B-V<1.0$. We refer to this combined sample as outlined in Carney et al. (2005) as the CL sample. This CL sample contains all binaries detected as spectroscopic binaries, visual binaries, or common proper motion pairs.

In Figure 15 we plot the binary fraction of stars on prograde and retrograde Galactic orbits, as shown in Figure 3 of Carney et al. (2005). All of the CL stars have $[\mathrm{Fe} / \mathrm{H}] \leq 0.0$. The CL distribution contains a small subset of metal-poor $[\mathrm{Fe} / \mathrm{H}] \leq-0.2$ stars from Ryan (1989) that has a one-third lower prograde binary fraction due to fewer observations. Thus, stars with metallicities between -0.2 and 0.0 have a higher binary fraction than the rest of the CL distribution. We make a small correction for this bias in the binary fraction in the range $-0.2<[\mathrm{Fe} / \mathrm{H}]<0.0$ by lowering the two highest metallicity prograde points of the CL distribution by $2 \%$.

We note an anticorrelation between the binary fraction and metallicity for the $-1.3<[\mathrm{Fe} / \mathrm{H}]<0.0$ range of prograde disk stars of the CL distribution, as shown in Figure 15. We find that the linear best fit to this anticorrelation has a gradient of $a=$ $-0.07 \pm 0.06$, and the nonlinear best fit has $\alpha=-0.12 \pm 0.09$, which are both significant at slightly above the $1 \sigma$ level. For consistency, we exclude the two lowest metallicity points from this best fit, so that we analyze the same region of metallicity as our samples and the GC sample, and because these two low-metallicity 

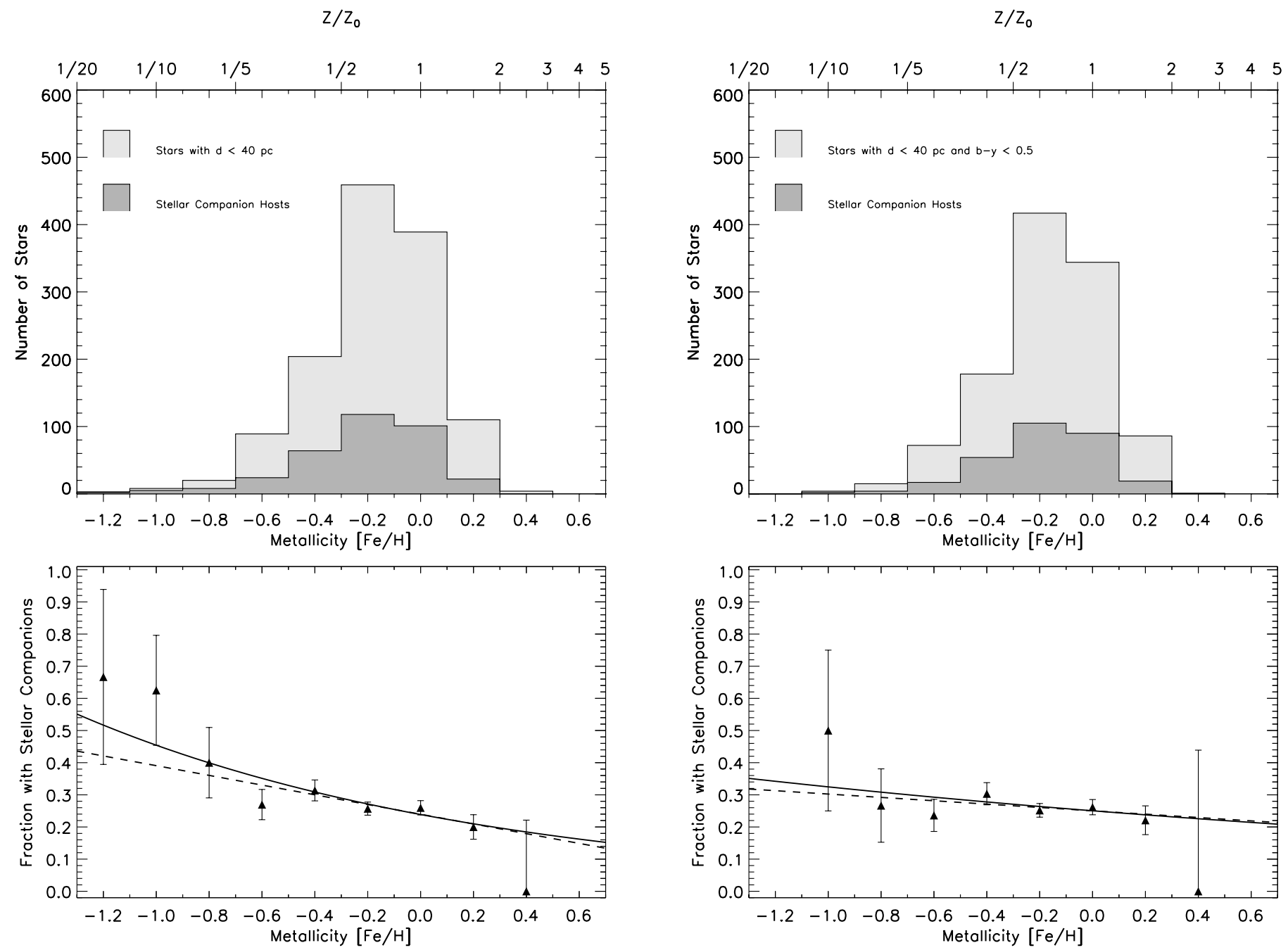

FIG. 12.-Histogram of stars in the complete volume-limited GC sample $(d<$ $40 \mathrm{pc}$ ). We exclude those stars with $b-y<0.3$ so that the spectral type range becomes F7-K3 and thus similar to that of our sample. We include only those stars that have between 2 and 10 radial velocity measurements with the CORAVEL spectrograph. We find an anticorrelation between binarity and host metallicity, as shown by the linear and nonlinear best fits represented by the dashed and solid lines, respectively.

points will probably contain a significant fraction of halo stars. The average binary fraction is $P_{\text {avg }}=26 \%$ for the disk-dominated part of the prograde CL distribution. Carney et al. (2005) found no correlation between binarity and host metallicity for the retrograde halo stars.

We overplot our $d<25 \mathrm{pc}$ binary fraction (from Fig. 4), along with the GC $d<40 \mathrm{pc}$ binary fraction (from Fig. 12), onto the prograde CL sample in Figure 15. All three of these samples have different binary period ranges and levels of completeness. We scale our sample and the GC sample to the size of the Carney et al. (2005) sample by scaling the distributions to contain the same number of binary stars at solar metallicity. The most metal-poor point in our close binary distribution is scaled above $100 \%$; hence we set this point to $100 \%$. The combined three-sample distribution shows an anticorrelation between binarity and metallicity. The normalized linear best fit to this is $a^{\prime}=(-0.10 \pm 0.03) / 25.7 \%=-0.39 \pm$ 0.12 , and the nonlinear best fit is $\alpha=-0.22 \pm 0.05$, which are both significant at or above the $\sim 3 \sigma$ level (see last row of Table 3 ). This combined result is our best estimate and indicates a strong anticorrelation between stellar companions and metallicity for $[\mathrm{Fe} / \mathrm{H}]>-1.3$.

Fig. 13.- Same as Fig. 12, but only for the FG dwarfs in the GC sample of stars $(d<40 \mathrm{pc})$. We define FG dwarfs as those with $b-y<0.5(B-V \lesssim$ 0.75 ). We find only a marginal anticorrelation between binarity and host metallicity, as shown by the linear best fit with gradient $a=-0.05 \pm 0.06$ and the nonlinear best fit with $\alpha=-0.11 \pm 0.10$. Using $P_{\text {avg }}=26.1 \%$, the scaled linear gradient $a^{\prime}=(-0.05 \pm 0.06) / 26.1 \%=-0.2 \pm 0.2$.

\subsection{Discussion}

We examine our results in terms of Galactic populations by determining the most likely population membership (halo, thick, or thin disk) for each star in the GC sample using the method outlined in the Appendix and then plotting them in the Galactic tangential velocity $V$-metallicity $[\mathrm{Fe} / \mathrm{H}]$ plane as in Figure 16. We use red points for the thin-disk stars, green points for the thick-disk stars, and a blue point for the single halo star. The kinematically unbiased GC sample contains mostly thin-disk stars. Excluding the one halo star in the GC sample, stars with $[\mathrm{Fe} / \mathrm{H}] \lesssim-0.9$ belong to the thick disk, and stars with $[\mathrm{Fe} / \mathrm{H}] \gtrsim-0.1$ belong to the thin disk. The region $-0.9 \gtrsim[\mathrm{Fe} / \mathrm{H}] \gtrsim-0.1$ contains a combination of both thick- and thin-disk stars.

We also plot these thick- and thin-disk stars as separate histograms in metallicity in Figure 17. In the region $[\mathrm{Fe} / \mathrm{H}] \lesssim-0.9$, which contains only thick-disk stars, we find that the binary fraction is approximately twice as large as for the region $[\mathrm{Fe} / \mathrm{H}] \gtrsim$ -0.1 , which contains only thin-disk stars. In both of these single-population regions, the binary fraction also appears to be approximately independent of metallicity. While our purely probabilistic method of assigning the stars in the GC sample to 

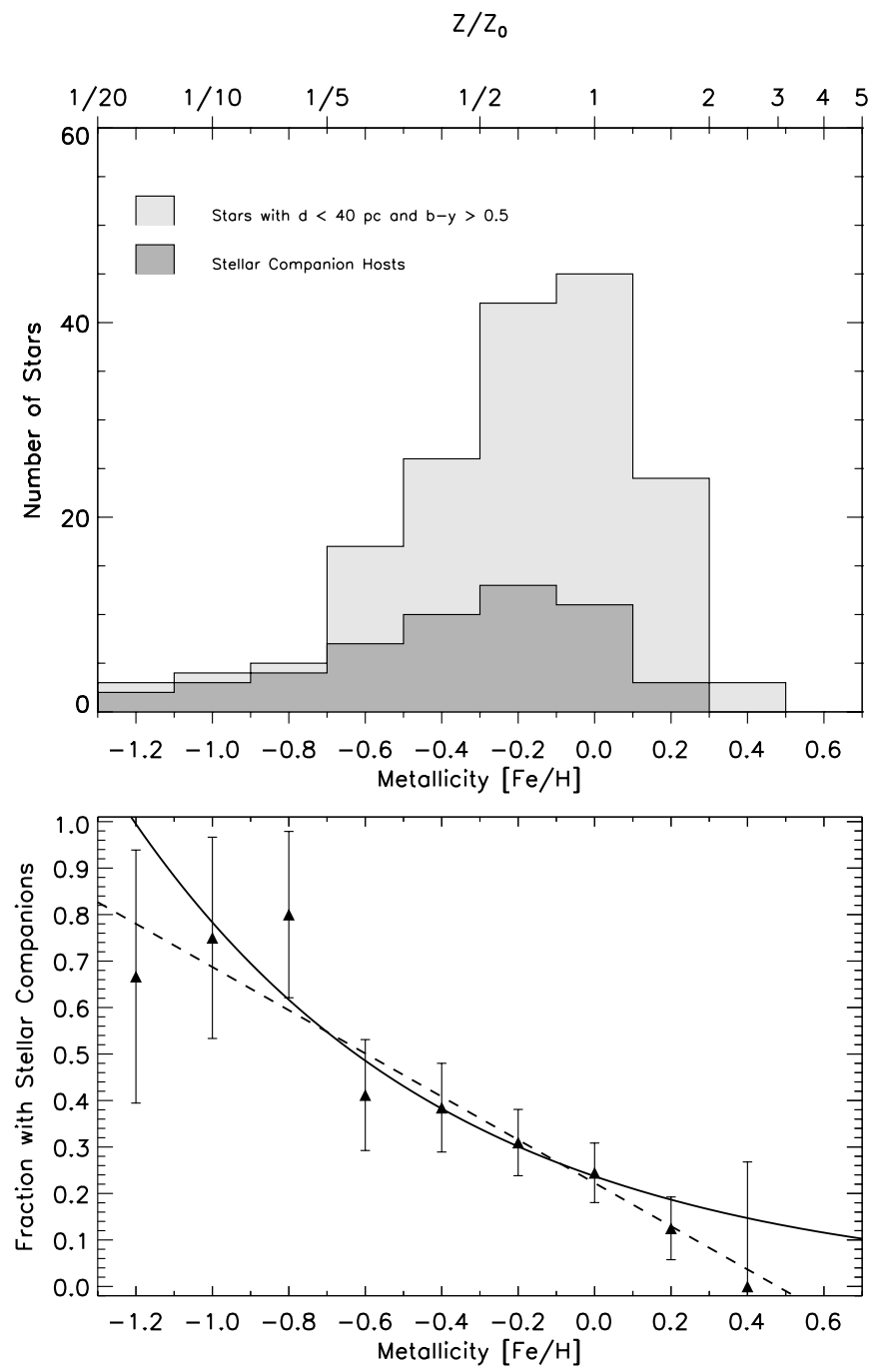

FIG. 14.- Same as Fig. 12, but only for the $\mathrm{K}$ dwarfs in the GC sample of stars $(d<40 \mathrm{pc})$. We define $\mathrm{K}$ dwarfs as those with $b-y>0.5(B-V \gtrsim 0.75)$. We find a very strong anticorrelation between binarity and host metallicity, as shown by the linear best fit with gradient $a=-0.46 \pm 0.10$. The nonlinear best fit is $\alpha=-0.52 \pm 0.11$. The scaled linear gradient $a^{\prime}=(-0.46 \pm 0.10) / 32.0 \%=$ $-1.4 \pm 0.3$. Comparing this plot with Fig. 13 suggests that the anticorrelation between binarity and host metallicity is stronger for redder stars.

Galactic populations is useful for determining the general regions of parameter space that the individual populations occupy, it is not precise enough to show exactly which stars belong to which population. This is especially true for the regions of parameter space that have large overlaps, such as that between the thick- and thindisk stars in Figure 16. Thus, the thin- and thick-disk binary fractions in the interval $-0.9 \lesssim[\mathrm{Fe} / \mathrm{H}] \lesssim-0.1$ are probably mixtures. We suspect that the thin- and thick-disk binary fractions in this overlap region will remain at the same levels as found for the nonoverlapping regions. The anticorrelation between binarity and metallicity in the $-0.9 \lesssim[\mathrm{Fe} / \mathrm{H}] \lesssim-0.1$ range may be due to this overlap between higher binarity thick-disk stars and lower binarity thin-disk stars.

We now partition the Galactic tangential velocity $V$-metallicity $[\mathrm{Fe} / \mathrm{H}]$ parameter space into four quadrants. We split the $V$-parameter space into those stars on prograde Galactic orbits (P) and those on retrograde Galactic orbits $(\mathrm{R})$. We split the $[\mathrm{Fe} / \mathrm{H}]$ parameter space into those stars that are metal-rich (r), with $[\mathrm{Fe} / \mathrm{H}] \gtrsim-0.9$, and those that are metal-poor $(\mathrm{p})$, with $[\mathrm{Fe} / \mathrm{H}] \lesssim$ -0.9 . We then label these quadrants by the direction of Galactic

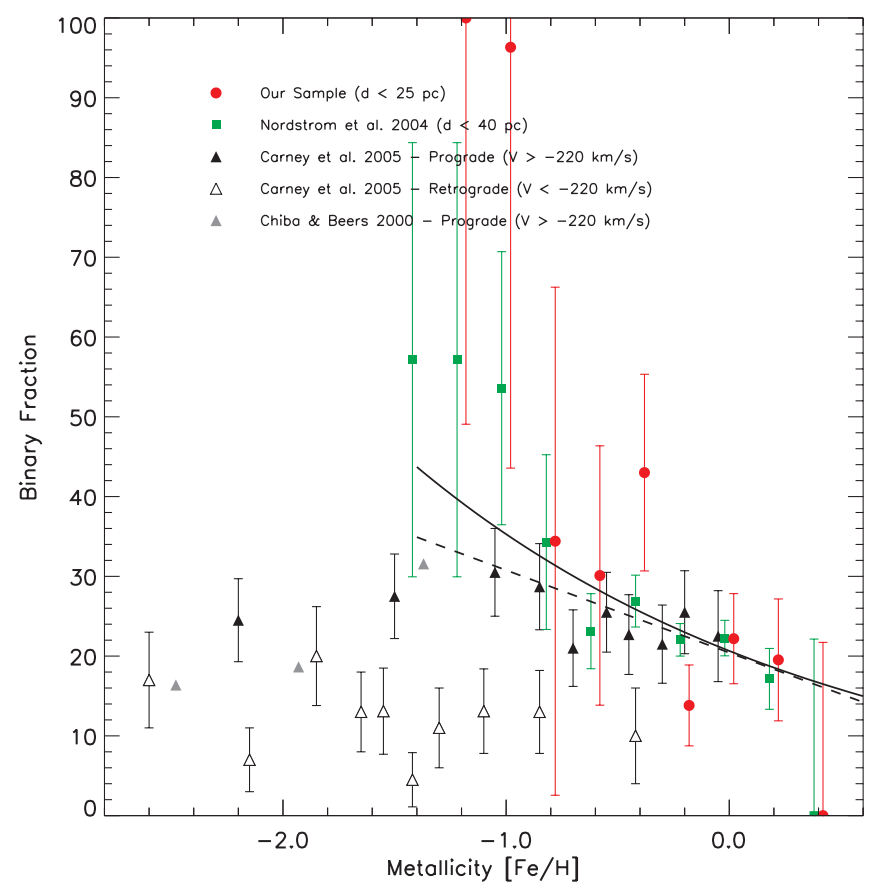

FIG. 15.-Plot adapted from Fig. 3 of Carney et al. (2005). The black triangles are the points from the CL sample of proper-motion stars with prograde Galactic tangential velocities. We overplot the binary fraction as a function of host metallicity for our close $(d<25 \mathrm{pc}) \mathrm{F} 7-\mathrm{K} 3$ sample (Fig. 4$)$ with red circles, and the green squares are from the volume-limited GC sample $(d<40 \mathrm{pc})$ for F7-K3 stars (Fig. 12). The three samples contain different average binary fractions because the period range and the levels of completeness of the stellar companions vary between the samples, as discussed in the text. We normalize the distributions by scaling our sample and the GC sample so that they contain the same fraction of binary stars as the sample of Carney et al. (2005) at $[\mathrm{Fe} / \mathrm{H}]=0$. The linear and nonlinear best fits to the three samples combined are shown as dashed and solid lines, respectively.

orbital motion followed by the range in metallicity, or $\mathrm{Pp}, \mathrm{Pr}, \mathrm{Rr}$, and Rp, as shown in Figure 16. We now assume that the Pp quadrant contains a mixture of halo and thick-disk stars, that the $\mathrm{Pr}$ quadrant contains a mixture of thin- and thick-disk stars, and that the $\mathrm{Rp}$ and $\mathrm{Rr}$ quadrants contain only halo stars.

The combined anticorrelation between binarity and metallicity shown in Figure 15, that all three samples appear to have in common, is predominantly shown in the Pr quadrant of $V-[\mathrm{Fe} / \mathrm{H}]$ parameter space, which contains a mixture of thick- and thin-disk stars. As discussed above, this anticorrelation may be due to the overlap of high-binarity thick-disk stars and lower binarity thindisk stars.

While Latham et al. (2002) suggest that the halo and disk populations have the same binary fraction, Carney et al. (2005) find lower binarity in retrograde stars. As shown in Figure 15, there is a clear difference of about a factor of 2 in the region $[\mathrm{Fe} / \mathrm{H}] \gtrsim$ -0.9 between the binary fractions of prograde disk stars and retrograde halo stars ( $\mathrm{Pr}$ and $\mathrm{Rr}$, respectively). All the retrograde halo stars appear to have the same binary fraction (quadrants $\mathrm{Rr}$ and $\mathrm{Rp}$ ). The Pp quadrant contains prograde halo stars and has a $\sim 2$ times higher binary fraction than the quadrants containing retrograde halo stars. However, the Pp quadrant also contains thickdisk stars in addition to prograde halo stars.

We propose that the $\mathrm{Pp}$ region, $[\mathrm{Fe} / \mathrm{H}] \lesssim-0.9$, for stars on prograde Galactic orbits contains a mixture of low-binarity halo stars and high-binarity thick-disk stars. In Figure 15 at $[\mathrm{Fe} / \mathrm{H}] \sim$ -0.9 , our close sample $(d<25 \mathrm{pc})$ and the GC sample $(d<$ $40 \mathrm{pc}$ ) start to diverge from the data points of the CL survey. This observed divergence may be due to the CL survey being 


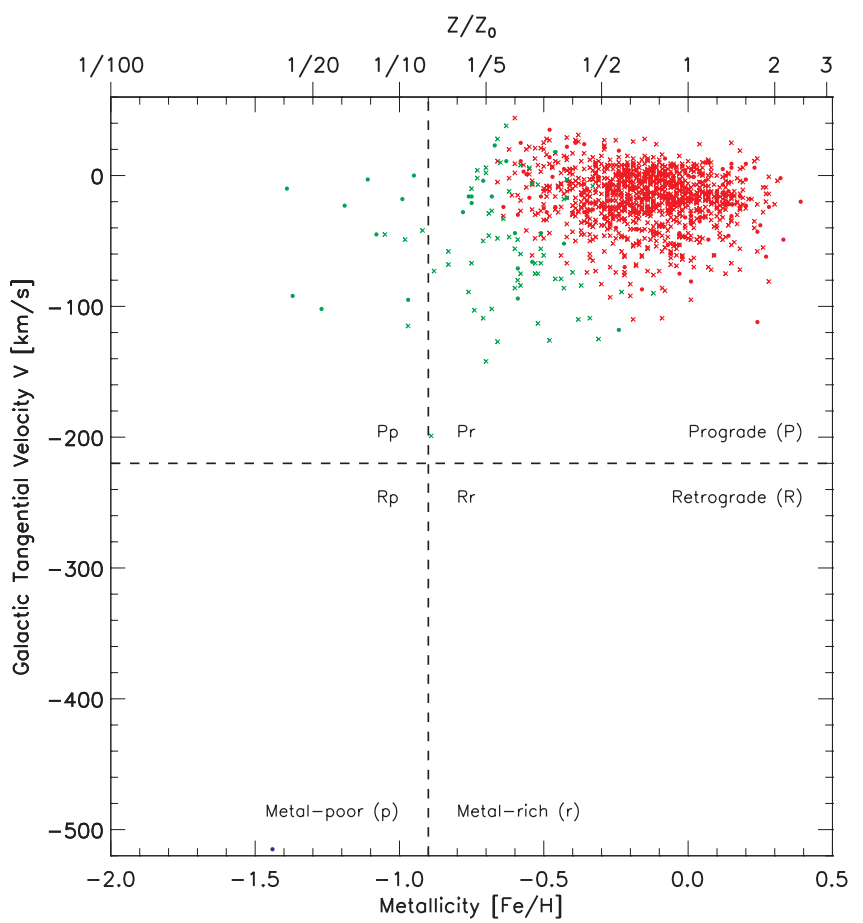

FIG. 16.-We plot tangential Galactic velocity $V$ as a function of metallicity $[\mathrm{Fe} / \mathrm{H}]$ for the kinematically unbiased GC sample $(d<40 \mathrm{pc})$. We use a probabilistic method to assign the stars in the GC sample to the three Galactic populations (halo, thick, and thin disks), as discussed in the Appendix. Red points represent thin disk stars, green points represent thick-disk stars, and the blue point represents the single halo star in the sample at $V<-500 \mathrm{~km} \mathrm{~s}^{-1}$. Crosses represent FG spectral type stars and circles, $\mathrm{K}$ stars. The ratio of thick/thin disk stars is $\sim 3$ times higher for $\mathrm{K}$ stars than for FG stars.

composed of high proper motion stars and consequently a higher fraction of prograde halo stars compared to thick-disk stars than the kinematically unbiased GC sample and our relatively kinematically unbiased sample, where thick-disk stars probably numerically dominate over halo stars.

Using a kinematically unbiased sample, Chiba \& Beers (2000) report for the three regions $-1.0>[\mathrm{Fe} / \mathrm{H}]>-1.7,-1.7>$ $[\mathrm{Fe} / \mathrm{H}]>-2.2$, and $-2.2>[\mathrm{Fe} / \mathrm{H}]$ that the fraction of stars that belong to the thick disk are $29 \%, 8 \%$ and $5 \%$, respectively, with the rest belonging to the halo. We restrict these thick-disk fraction estimates to stars only on prograde orbits by assuming that all of the thick-disk stars are on prograde orbits and that half of the halo stars are prograde and the other half are retrograde. Thus, the fraction of prograde stars that are thick-disk stars is $45 \%, 15 \%$, and $10 \%$ for the three metallicity regions, respectively.

Using these three prograde-restricted thick disk/halo ratios reported in Chiba \& Beers (2000), combined with the observed binary fraction for the thick-disk (55\%) and halo (12\%) stars in Figure 15, in the Pp quadrant we can test the proposal that the two lowest metallicity prograde points from Carney et al. (2005) contain a mixture of low-binarity halo stars and high-binarity thick-disk stars. We plot the three estimated mixed thick-disk/ halo binary fraction points as gray triangles in Figure 15 . We note that they are consistent with the two prograde Carney et al. (2005) points, thus supporting our proposal that the Pp quadrant contains a mixture of low-binarity halo stars and high-binarity thick-disk stars. These mixed thick disk/halo points also show a correlation between the presence of stellar companions and metallicity for stars in the $\mathrm{Pp}$ region.

Our results suggest that thick-disk stars have a higher binary fraction than thin-disk stars, which in turn have a higher binary
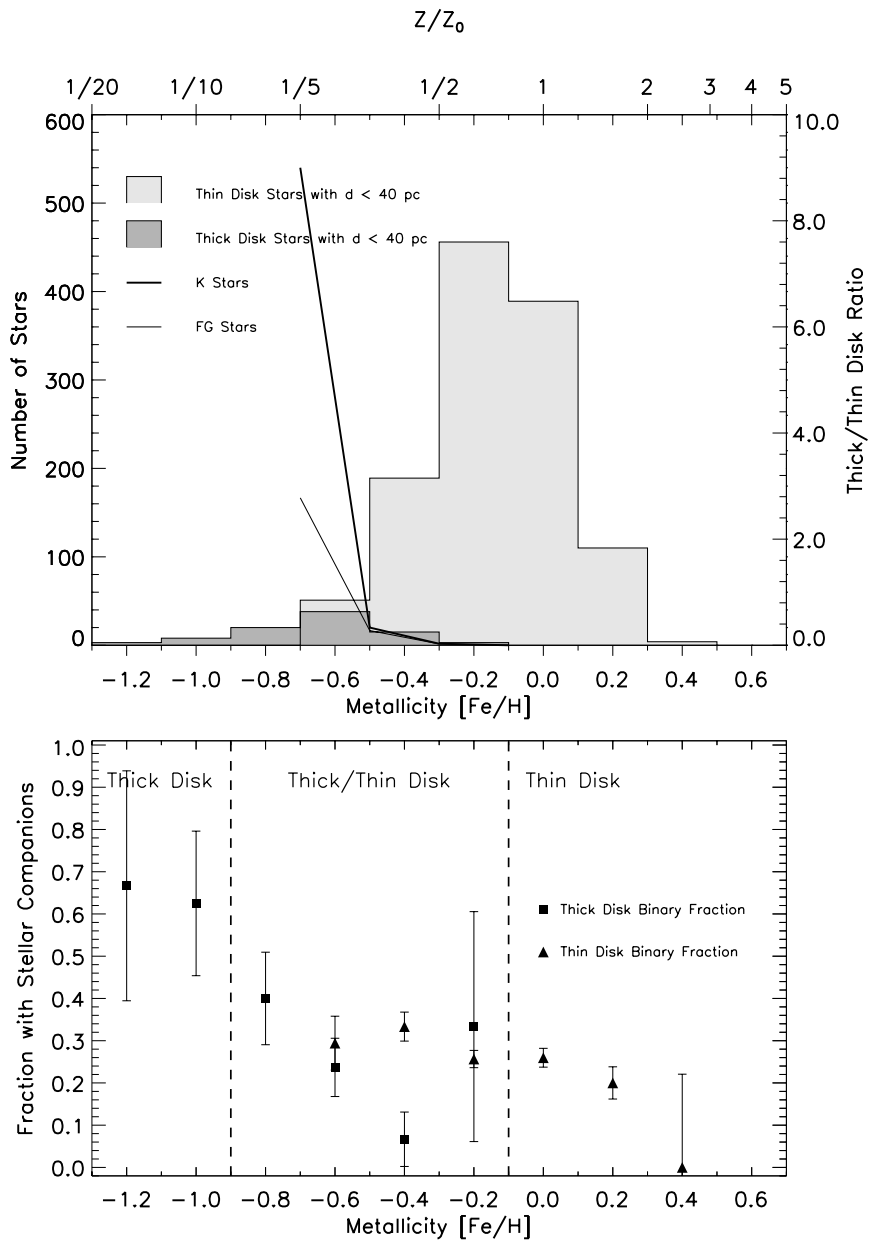

FIG. 17.- Histogram of the stars in Fig. 16 suspected of belonging to the thick disk and the thin disk in the GC sample $(d<40 \mathrm{pc})$. Note the difference by a factor $\sim 2$ between the higher binary fraction thick-disk stars and the lower binary fraction thin-disk stars. We note that the $\mathrm{K}$ star distribution contains a higher ratio of thick-disk stars than the FG star distribution in the thick/thin disk overlap region.

fraction than halo stars. Thus, for stars on prograde Galactic orbits, we observe an anticorrelation between binarity and metallicity for the region of metallicity $[\mathrm{Fe} / \mathrm{H}] \gtrsim-0.9$ that contains an overlap between the lower binarity, higher metallicity thin-disk stars and the higher binarity, lower metallicity thick-disk stars. We also find for stars on prograde Galactic orbits a correlation between binarity and metallicity for the range $[\mathrm{Fe} / \mathrm{H}] \lesssim-0.9$ that contains an overlap between the higher binarity, higher metallicity thick-disk stars and the lower binarity, lower metallicity halo stars.

\section{SUMMARY}

We examine the relationship between Sun-like (FGK dwarfs) host metallicity and the frequency of close companions (orbital period $<5 \mathrm{yr}$ ). We find a correlation at the $\sim 4 \sigma$ level between host metallicity and the presence of a planetary companion and an anticorrelation at the $\sim 2 \sigma$ level between host metallicity and the presence of a stellar companion. We find that the nonlinear best fit is $\alpha=2.2 \pm 0.5$ and $\alpha=-0.8 \pm 0.4$ for planetary and stellar companions, respectively (see Table 3 ).

Fischer \& Valenti (2005) also quantify the planet-metallicity correlation by fitting an exponential to a histogram in $[\mathrm{Fe} / \mathrm{H}]$. They find a best fit of $\alpha=2.0$. Our result of $\alpha=2.2 \pm 0.3$ is a slightly more positive correlation and is consistent with theirs. 
Our estimate is based on the average of the best fits to the metallicity data binned as a function of both $[\mathrm{Fe} / \mathrm{H}]$ and $Z / Z_{\odot}$. Larger bins tend to smooth out the steep turnup at high $[\mathrm{Fe} / \mathrm{H}]$ and may be responsible for their estimate being slightly lower.

We also analyze the sample of Nordström et al. (2004) and again find an anticorrelation between metallicity and close stellar companions for this larger period range. We also find that $\mathrm{K}$ dwarf host stars have a stronger anticorrelation between host metallicity and binarity than FG dwarf stars.

We compare our analysis with that of Carney et al. (2005) and find an alternative explanation for their reported binary frequency dichotomy between stars on prograde Galactic orbits with $[\mathrm{Fe} / \mathrm{H}] \lesssim 0$ compared to stars on retrograde Galactic orbits with $[\mathrm{Fe} / \mathrm{H}] \lesssim 0$. We propose that the region $[\mathrm{Fe} / \mathrm{H}] \lesssim-0.9$, for stars on prograde Galactic orbits, contains a mixture of lowbinarity halo stars and high-binarity thick-disk stars. Thick-disk stars appear to have a $\sim 2$ higher binary fraction compared to thindisk stars, which in turn have a $\sim 2$ higher binary fraction than halo stars.

While the ratio of thick/thin disk stars is $\sim 3$ times higher for $\mathrm{K}$ stars than for FG stars, we observe only a marginal difference in their distributions as a function of metallicity. In the region
$-0.9 \lesssim[\mathrm{Fe} / \mathrm{H}] \lesssim-0.1$, which we suspect contains a mixture of thick- and thin-disk stars, the $\mathrm{K}$ star distribution contains a higher ratio of thick-disk stars compared to the FG star distribution at a given metallicity. This difference is marginal, but can partially explain the kinematic and spectral type ( $\sim$ mass $)$ results.

Thus, for stars on prograde Galactic orbits, as we move from low metallicity to high metallicity we move through low-binarity halo stars to high-binarity thick-disk stars to medium-binarity thindisk stars. Since halo, thick-disk, and thin-disk stars are not discrete populations in metallicity and contain considerable overlap, as we go from low-metallicity to high-metallicity for prograde stars, we first observe a correlation between binarity and metallicity for the overlapping halo and thick-disk stars, and then an anticorrelation between binarity and metallicity for the overlapping thick- and thin-disk stars.

We would like to thank Johan Holmberg for his help in analyzing the Geneva sample and Chris Flynn, John Norris, Virginia Trimble, Richard Larson, Pavel Kroupa, and David Latham for helpful discussions.

\section{APPENDIX}

\section{PROBABILITY OF GALACTIC POPULATION MEMBERSHIP}

We use a method similar to that of Reddy et al. (2006) in assigning a probability to each star of being a member of the thin-disk, thick-disk, or halo populations. We assume that the GC sample is a mixture of the three populations. These populations are assumed to be represented by a Gaussian distribution for each of the three Galactic velocities $U, V$, and $W$ and for the metallicity [Fe/ $\mathrm{H}]$. The age dependence of the quantities for the thin disk is ignored. The equations establishing the probability that a star belongs to the thin disk $\left(P_{\text {thin }}\right)$, the thick disk $\left(P_{\text {thick }}\right)$, or the halo $\left(P_{\text {halo }}\right)$ are

$$
P_{\text {thin }}=f_{1} \frac{P_{1}}{P}, \quad P_{\text {thick }}=f_{2} \frac{P_{2}}{P}, \quad P_{\text {halo }}=f_{3} \frac{P_{3}}{P},
$$

where

$$
\begin{aligned}
P & =\sum f_{i} P_{i} \\
P_{i} & =C_{i} \exp \left[-\frac{U^{2}}{2 \sigma_{U_{i}}^{2}}-\frac{(V-\langle V\rangle)^{2}}{2 \sigma_{V_{i}}^{2}}-\frac{W^{2}}{2 \sigma_{W_{i}}^{2}}-\frac{([\mathrm{Fe} / \mathrm{H}]-\langle[\mathrm{Fe} / \mathrm{H}]\rangle)^{2}}{2 \sigma_{[\mathrm{Fe} / \mathrm{H}]_{i}}^{2}}\right], \\
C_{i} & =\frac{1}{\sigma_{U_{i}} \sigma_{V_{i}} \sigma_{W_{i}} \sigma_{[\mathrm{Fe} / \mathrm{H}]_{i}}} \quad(i=1,2,3) .
\end{aligned}
$$

Using the data in Table 4 taken from Robin et al. (2003), we compute the probabilities for stars in the GC sample. For each star, we assign it to the population (thin disk, thick disk, or halo) that has the highest probability. We plot the probable halo, thick-, and thindisk stars of the GC sample in Figure 16.

TABLE 4

Properties of the Three Stellar Populations

\begin{tabular}{crrrrrrr}
\hline \hline Component & $\sigma_{U}$ & $\langle V\rangle$ & $\sigma_{V}$ & $\sigma_{W}$ & $\langle[\mathrm{Fe} / \mathrm{H}]\rangle$ & $\sigma[\mathrm{Fe} / \mathrm{H}]$ & Fraction $f$ \\
\hline Thin disk .................. & 43 & -15 & 28 & 17 & -0.1 & 0.2 & 0.925 \\
Thick disk .................. & 67 & -53 & 51 & 42 & -0.8 & 0.3 & 0.070 \\
Halo........................ & 131 & -226 & 106 & 85 & -1.8 & 0.5 & 0.005 \\
\hline
\end{tabular}

NotE.-Data from Robin et al. (2003). 
Ammons, S. M., Robinson, S. E., Strader, J., Laughlin, G., Fischer, D., \& Wolf, A. 2006, ApJ, 638, 1004

Batten, A. H. 1973, Binary and Multiple Systems of Stars (Oxford: Pergamon) Bond, J. C., Tinney, C. G., Butler, P., Jones, H. R. A., Marcy, G. W., Penny, A. J., \& Carter, B. D. 2006, MNRAS, 370, 163

Carney, B. W., Angular, L. A., Latham, D. W., \& Laird, J. B. 2005, AJ, 129, 1886

Carney, B. W., \& Latham, D. W. 1987, AJ, 93, 116

Carney, B. W., Latham, D. W., Laird, J. B., \& Aguilar, L. A. 1994, AJ, 107, 2240

Cayrel de Strobel, G., Soubiran, C., \& Ralite, N. 2001, A\&A, 373, 159 (CdS)

Chiba, M., \& Beers, T. C. 2000, AJ, 119, 2843

Cox, A. N., ed. 2000, Allen's Astrophysical Quantities (4th ed.; New York: AIP)

Dall, T. H., Bruntt, H., \& Strassmeier, K. G. 2005, A\&A, 444, 573

Duquennoy, A., \& Mayor, M. 1991, A\&A, 248, 485

Fischer, D. A., \& Valenti, J. 2005, ApJ, 622, 1102

Giclas, H. L., Burnham, R., \& Thomas, N. G. 1971, Lowell Proper Motion Survey: Northern Hemisphere, The G Numbered Stars, 8991 Stars Fainter than Magnitude 8 with Motions $>0.26^{\prime \prime} \mathrm{yr}^{-1}$ (Flagstaff: Lowell Obs.)

Giclas, H. L., Burnham, R., Jr., \& Thomas, N. G. 1978, Lowell Obs. Bull., 8, 89

Gonzalez, G. 1997, MNRAS, 285, 403

Gonzalez, G., Laws, C., Tyagi, S., \& Reddy, B. E. 2001, AJ, 121, 432

Grether, D., \& Lineweaver, C. H. 2006, ApJ, 640, 1051

Gunn, J. E., \& Griffin, R. F. 1979, AJ, 84, 752

Hauck, B., \& Mermilliod, M. 1998, A\&AS, 129, 431

Jones, H. R. A., Butler, R. P., Marcy, G. W., Tinney, C. G., Penny, A. J., McCarthy, C., \& Carter, B. D. 2002, MNRAS, 337, 1170
Jones, H. R. A., Butler, R. P., Tinney, C. G., Marcy, G. W., Carter, B. D., Penny, A. J., McCarthy, C., \& Bailey, J. 2006, MNRAS, 369, 249

Kotoneva, E., Flynn, C., \& Jimenez, R. 2002, MNRAS, 335, 1147

Latham, D. W. 2004, in ASP Conf. Ser. 318, Spectroscopically and Spatially Resolving the Components of the Close Binary Stars, ed. R. W. Hilditch, H. Hensberge, \& K. Pavlovski (San Francisco: ASP), 276

Latham, D. W., Mazeh, T., Carney, B. W., McCrosky, R. E., Stefanik, R. P., \& Davis, R. J. 1988, AJ, 96, 567

Latham, D. W., Stefanik, R. P., Torres, G., Davis, R. J., Mazeh, T., Carney, B. W., Laird, J. B., \& Morse, J. A. 2002, AJ, 124, 1144

Laws, C., Gonzalez, G., Walker, K. M., Tyagi, S., Dodsworth, J., Snider, K., \& Suntzeff, N. B. 2003, AJ, 125, 2664

Lineweaver, C. H., \& Grether, D. 2003, ApJ, 598, 1350

Luyten, W. J. 1979, The NLTT Catalog, Vols. I and II (Minneapolis: Univ. Minnesota)

—. 1980, The NLTT Catalog, Vols. III and IV (Minneapolis: Univ. Minnesota)

Martell, S., \& Smith, G. H. 2004, PASP, 116, 920

Nordström, B., et al. 2004, A\&A, 418, 989

Reddy, B. E., Lambert, D. L., \& Allende Prieto, C. 2006, MNRAS, 367, 1329

Reid, I. N. 2002, PASP, 114, 306

Robin, A. C., Reylé, C., Derrière, S., \& Picaud, S. 2003, A\&A, 409, 523

Ryan, S. G. 1989, AJ, 98, 1693

Santos, N. C., Israelian, G., \& Mayor, M. 2004, A\&A, 415, 1153

Santos, N. C., Israelian, G., Mayor, M., Bento, J. P., Almeida, P. C., Sousa, S. G., \& Ecuvillon, A. 2005, A\&A, 437, 1127

Valenti, J. A., \& Fischer, D. A. 2005, ApJS, 159, 141 